\title{
Mejores Pensiones con Modelos Matemáticos Actuariales más Representativos ${ }^{1}$
}

\section{Best Pensions with Most Representative Actuarial Mathematical Models}

\author{
José E. Cavero Vicentelo² \\ Miguel A. Pinglo Ramírez ${ }^{3}$
}

\section{RESUMEN}

En el Perú y en el mundo se observa gran evolución científica, tecnológica, de conocimientos, de acumulación de capitales, etc. frente a una cada vez mayor desigualdad, caída en la proporción de las remuneraciones respecto el ingreso, se eluden impuestos en paraísos fiscales, reemplazo del recurso humano por el tecnológico, etc. y se estiman pensiones generalmente pequeñas, acentuando la desigualdad,

1 Se investiga proponiendo mayor representatividad en los modelos, incorporando esperanzas de vida individualizadas, según tesis: "Modelos matemáticos actuariales y fondos previsionales pensionarios: rentas vitalicias hasta 110 años o hasta cuando el pensionista fallezca de manera más probable".

2 Ingeniero Economista por la Universidad Nacional de Ingeniería. Actuario Matemático por la Universidad Nacional Mayor de San Marcos y la Organización Iberoamericana de Seguridad Social. Actualmente es Actuario independiente y Profesor universitario. (e-mail: jcavero777@outlook.com), teléfono móvil 992445251

3 Doctor en Gestión Económica Global y Doctorado en Administración por la Universidad Nacional Mayor de San Marcos. Master en Ciencias de la Gestión por la Universidad de Montesquieu IV Bordeaux - Francia, Máster of Bussines Administration por la Universidad San Ignacio de Loyola. Economista especializado en Gestión de Proyectos y Negociaciones Transfronterizas por la University of Tampa - USA. Actualmente es Profesor Investigador adscrito al Instituto de Investigaciones de la Facultad de Ciencias Económicas de la Universidad Nacional Mayor de San Marcos.(e-mail: mpinglor@ unmsm.edu.pe)

(C) Los autores. Este artículo es publicado por Pensamiento Crítico de la Facultad de Ciencias Económicas, Universidad Nacional Mayor de San Marcos. Este es un artículo de acceso abierto, distribuido bajo los términos de la licencia Creative Commons Atribucion - No Comercia_Compartir Igual 4.0 Internacional. (http:// creativecommons.org/licenses/by-nc-sa/4.0/) que permite el uso no comercial, distribución y reproducción en cualquier medio, siempre que la obra original sea debidamente citada. 
pensando que en el Perú por ejemplo, una persona de 65 años vivirá 45 años más hasta los 110 años.

Se propone mejorar la pensión, al interrelacionar los conceptos de modelo y equilibrio actuarial con la esperanza de vida al momento de adquirir derecho, logrando mayor representatividad. La edad más probable del fallecimiento la estima la esperanza de vida, definida como el número de años que en promedio aún le faltan vivir a la persona según su edad y sexo al cese o adquirir derecho, con resultados de hasta más del 25\%, pues con las tablas vigentes una persona varón o mujer de 65 años vivirá más probablemente 17 ó 22 años más y no 45 años más.

Palabras clave: Modelo; modelo actuarial; esperanza de vida; producto pensionario; desigualdad.

JEL: G, G22, G23

\section{ABSTRACT}

In Peru and in the world there is a great scientific, technological, knowledge, accumulation of capital, etc. evolution. in the face of an increasing inequality, a fall in the proportion of salaries with respect to income, tax evasion is avoided in tax havens, replacement of human resources by technology, etc. and generally small pensions are estimated, accentuating inequality, thinking that in Peru for example, a person of 65 years will live 45 years more until 110 years.

It is proposed to improve the pension, by interrelated model concepts and actuarial balance with life expectancy at the time of acquiring the right, achieving greater representation. The most probable age of death is estimated by life expectancy, defined as the number of years that, on average, the person still has to live according to age and sex upon cessation or acquisition of rights, with results of up to more than $25 \%$. with the current tables, a 65-year-old man or woman will probably live 17 or 22 years more and not 45 years more.

Keywords: Model; Actuarial model; Life expectancy; Pension product and Inequality.

\section{Introducción}

Ante la cada vez mayor desigualdad, explicada con cifras en los siguientes párrafos, se presenta una alternativa para estimar pensiones partiendo de modelos actuariales que incorporen la esperanza de vida de cada pensionista, obteniendo con ello no solo modelos más representativos sino mayores montos en las rentas vitalicias.

No solo en el Perú sino en el mundo, según el Informe del Fondo Monetario Internacional (FMI) del año 2017, generalmente los más 
ricos concentran cada vez más riqueza, situación que genera una mayor desigualdad y a la par una caída en la proporción de las remuneraciones respecto al ingreso. Se busca favorecer a los nuevos pensionistas de invalidez, viudez, ascendencia y jubilación, los que sumando los registrados en el Sistema Privado de Pensiones (SPP) y aquellos que pertenecen al Sistema Nacional de Pensiones (SNP), cada mes superan en promedio los dos mil nuevos pensionistas, sin contar otros regímenes de contratación o beneficios mencionados, entre otros, en la obra de Martín Fajardo por ejemplo incluida en la bibliografía. Según el FMI, en su Informe de Perspectivas de la Economía Mundial de abril del 2017, en las últimas tres o cuatro décadas, la participación de los trabajadores en el ingreso nacional viene disminuyendo incluso en los países avanzados del $54 \%$ al $40 \%$, en las economías emergentes y en desarrollo del $50 \%$ al 38\% y en el Perú, según el Instituto Nacional de Estadística e Informática (INEI): PBI según Tipo de Ingreso, 2016, la caída de la remuneración respecto el $\mathrm{PBI}$, pasa del $47 \%$ al $20 \%$, aunque con el ingreso de los independientes se llegaría a una proporción cercana al 31\%.Tal situación no solo compromete el dinamismo económico por el lado de los ingresos, sino que incide también en mayor frustración, mermando la salud mental y física, en el comportamiento y bajo rendimiento del capital humano e incluso en la delincuencia, en nuevas formas de corrupción, en la pérdida de credibilidad en líderes y formas democráticas, etc.

Se desea demostrar la viabilidad de pagar pensiones más altas en el SPP, empleando nuevos modelos matemáticos actuariales propiciando mejor redistribución de la renta, inclusión y contribuyendo con el proceso de desarrollo económico ante una generalizada desigualdad. Es necesario verificar la elaboración actual de los modelos vigentes y proponer modelos mejorados más representativos que incluyan esperanzas de vida individualizadas y tablas de mortalidad adecuadas a la población en estudio.

\subsection{Justificación y limitaciones}

El modelo propuesto, no solo será más representativo sino que beneficiaría a miles de afiliados al SPP e incluso a los de los fondos administrados por la Oficina de Normalización Previsional (ONP), a la Caja de Pensiones Militar Policial, la Caja del Pescador y otros fondos más pequeños, en la medida 
que se obtenga equilibrio actuarial previo en dichos regímenes públicos, lo cual es una limitación, teniendo en cuenta la situación deficitaria actual la que en última instancia es financiada por la caja fiscal, desvirtuando el objetivo original de creación de dichos fondos, referido al alivio de la obligación previsional del Estado.

Las empresas privadas de seguros al aumentar las pensiones con lo propuesto, disminuirían en algo sus utilidades probablemente solo en un principio, ya que los mayores desembolsos no se verían como un gasto sino más bien como una inversión en la calidad del producto pensionario, el que atraería a más afiliados conjuntamente con las últimas normas referidas al retiro a los 65 años de hasta 95.5\% del fondo y en cualquier momento del 25\% para vivienda propia, según Ley 30425 del 15 de abril del 2016, circunstancias que evaluadas en su conjunto debieran de contribuir en nuestro camino hacia una sociedad más desarrollada, solidaria y justa.

En el esperado mejor escenario hacia el largo plazo, no solo las tasas internas de retorno (TIR) de las empresas disminuirían sino también las tasas de inflación, las de devaluación, las tasas de crecimiento del PBI, etc., debido a los progresivos logros y a los mejores niveles de estabilidad y a menores niveles de riesgo esperados de la sociedad en su conjunto.

\subsection{Mejoramiento de la naturaleza previsional}

Con las mejores pensiones, no todos los afiliados querrían retirar hasta el 95.5\% de sus fondos según su nivel de aversión al riesgo, toda vez que por la cercanía a los 65 años, podría generar una posición más defensiva y de menor riesgo percibido con la posible mayor pensión, disminuyendo así el riesgo de afectar la naturaleza previsional de su fondo y mejorando su posición para enfrentar mejor la pobreza y la desigualdad.

Muchas nuevas Pequeñas y Medianas Empresas (PYME) dejan de existir durante los primeros tres años, siendo la principal razón la baja rentabilidad, generada más allá de las políticas y ciclos en la economía y la edad del inversionista, por déficits en la profesionalización, en la calidad del producto, en la estandarización de las operaciones, en la gestión de los trabajadores, en el control de insumos y proveedores, en la planificación, 
en el análisis de resultados, en el liderazgo de los dueños, por el mal uso del financiamiento, por no reinversiones adecuadas, por no escuchar a sus clientes, etc.

En el Perú, según el INEI para su Informe de Demografía Empresarial en el Perú, en el año 2016 surgen o se generan 262,837 altas, pero también se dan bajas por 190,816 empresas, por lo que se requiere insistir en una asesoría muy profesional a los pensionistas que opten por el retiro de su hasta $95.5 \%$.

\section{Marco teórico:}

La casuística presentada emplea datos reales pues proviene de los modelos vigentes que constituyen fuentes secundarias en cuanto son fuentes fidedignas, verídicas, aceptadas en instituciones públicas y privadas en el país y en el extranjero y elaboradas por especialistas en los temas, habiéndose desarrollado todas las posibilidades por edades y sexos.

\subsection{La desigualdad aumenta en el mundo y en el Perú también}

Si bien en general la pobreza disminuye (excepto el último año que aumentó) la desigualdad aumenta de manera concomitante con la menor participación del trabajo en los ingresos. Sin embargo, muchos críticos de la actual metodología de medición de la pobreza en el INEI, piensan que en nuestro país no son uno de cada cinco los pobres sino más bien uno de cada tres si se considerase para medir la pobreza no solo el aspecto monetario por el cual con más de S/338.- por mes ya no se es pobre y con más de S/183.- mensuales no se es pobre extremo (según su Informe del 2017); si se considerase el enfoque multidimensional o multivariable que incluye carencias objetivas de acceso a la salud, educación, vivienda, agua y desagüe, nutrición, etc., los pobres serían efectivamente uno de cada tres en el Perú, pues los mencionados S/338.- del enfoque solamente monetario quedarían muy cortos.

Cada vez es más preocupante la menor participación de las remuneraciones en el ingreso, pero también preocupa el aún muy alto nivel de desigualdad medido con el coeficiente de Gini. 
Según el Informe de Desarrollo Humano de Naciones Unidas, 2005, un coeficiente Gini con un nivel superior al 0.4 es alarmante pues genera muchas tensiones y malestar; a nivel global se ubica en más del 0.5, con cifras del orden del 0.2 en los países más avanzados y menos desiguales y con cifras superiores al 0.6 en los más atrasados y desiguales. En el Perú si bien se ha reducido por el crecimiento de los últimos años, aún se encuentra en un nivel cercano al alarmante $0.4 \mathrm{y}$ con el riesgo de no solo no bajar sino de incrementarse de no ponderarse más el aspecto de desarrollo respecto del actual no óptimo crecimiento.

\subsection{Muy bajas probabilidades de llegar a los 110 años}

En elSPP se estiman montos de rentas vitalicias en las compañías de seguros privadas bajo la consideración de que las personas podrían llegar a cobrar sus rentas o pensiones hasta los 110 años, lo cual es cierto pero muy poco probable de que así ocurra. De acuerdo a la tabla de mortalidad vigente de afiliados en actividad, la renta vitalicia para hombres (RV Hombres) señala que la probabilidad que un varón en actividad entre 20 y 70 años de edad, llegue con vida a los 110 años es bajísima: 0.03\%aproximadamente, o lo que es lo mismo 3 casos en diez mil (Cuadro № 01) y, lo que no se hace es trabajar en las estimaciones de los montos de las rentas vitalicias con el criterio de lo que va a ocurrir de manera más probable, con lo cual dicha persona solo va a vivir según lo defina su correspondiente esperanza de vida a determinada edad y sexo.

Las empresas aseguradoras privadas, asumen una posición conservadora pagando una menor pensión que obviamente cubre a la aseguradora, pero definiendo una menor renta para el pensionista.

La propuesta del presente trabajo, empezó con las estimaciones de las esperanzas de vida, luego con la elaboración de los modelos vigentes, después con los modelos propuestos y una serie de casos demostrativos.

\subsection{Modelo en general y modelo actuarial en particular}

Es importante recordar algo del concepto de modelo en general y modelo actuarial en particular, conceptos que se amplían en la tesis que es base del presente artículo: "Modelos matemáticos actuariales y fondos previsionales pensionarios: rentas vitalicias hasta 110 años o hasta cuando el pensionista fallezca de manera más probable". 
Un modelo, resumiendo, es una representación de un sistema o fenómeno bajo estudio, del cual deben representarse sus características básicas u originales y de funcionamiento; es decir, un modelo es una versión a escala diferente de un determinado fenómeno o sistema, pero que debe mantener los rasgos y características del sistema bajo estudio o problema analizado.

El modelo actuarial propuesto, al incorporar las esperanzas de vida individualizadas representa mejor la realidad, pues resulta consistente con la acepción más amplia de modelo actuarial, referida aque el modelo es obtenido a través de la utilización de metodologías científicas convenientes y de un grupo generalmente interdisciplinario, con el objeto de representar las diferentes relaciones funcionales complejas de un sistema o subsistema de Seguro Social o Seguro Privado, diagnosticando sus características básicas y de funcionamiento presentes y estimando las futuras, delimitando las denominadas "bases técnicas" que nos permitan sustentar un plan o sistema de previsión previamente definido, para así de esta manera proporcionar una base cuantitativa en la toma de decisiones y garantizar su equilibrio.

El grupo interdisciplinario es necesario pues genera un trabajo conjunto de hombres de ciencia de varias disciplinas en una investigación o problema, con lo cual se aumentan y enriquecen los enfoques posibles para resolverlo.

El modelo actuarial pasará de cualitativo a cuantitativo, será construido a la medida y circunstancias de un sistema cambiante y por lo tanto también será dinámico, probabilístico, de optimización y simulación.

Las "bases técnicas" están referidas a las diferentes variables que intervienen en la labor actuarial, es decir las bases biométricas, demográficas, económicas y financieras, además de las consideraciones respecto del entorno del sistema y el marco legal.

\section{Metodología}

En el presente trabajo cuantitativo actuarial con implicancias económicas financieras y ddebido a que las aplicaciones vigentes constituyen fuentes secundarias en cuanto son fuentes fidedignas, verídicas, aceptadas en 
instituciones públicas y privadas en el país y en el extranjero y elaboradas por especialistas en los temas, entonces se verifica que las normas que las acompañan con las principales características del SPP que se refieren en la tesis mencionada en el Marco Legal del Anexo № 01 parte 8, especifican que las estimaciones de los montos de pensiones a pagar deben efectuarse hasta el límite de la tablas vigentes bajo el criterio de rentas vitalicias de vida entera, por lo que la totalidad de las actuales aplicaciones constituyen una base poblacional bastante completa y se obvia en principio el empleo de fórmulas habituales para encontrar tamaños de muestra, ya que se desarrollan todas las posibilidades por edades y sexos y no solo a partir de los 65 años, que es la edad actual de jubilación, sino también para los casos de jubilación anticipada con 12 meses o más de desempleo a partir de los 55 años para los hombres y a partir de los 50 años para las mujeres, denominado Régimen de jubilación Anticipada por Desempleo (REJA).

Así mismo se considera que la investigación no experimental es aquella que se realiza sin manipular deliberadamente variables, es decir, es aquella investigación donde no hacemos variar intencionalmente las variables independientes y lo que hacemos en la investigación no experimental es observar fenómenos tal y como se dan en su contexto natural, para después analizarlos; por tanto esta concepción es consistente con la definición de modelo en general y en particular con el concepto de modelo matemático actuarial, tal como se menciona en el párrafo anterior y se amplía en la tesis respectiva, ya que todo modelo debe representar de la mejor manera a partir de un sistema dinámico la interrelación de las variables involucradas.

Por lo tanto, en el presente trabajo, efectuamos básicamente la investigación no experimental, porque lo que se efectúa no es hacer variar intencionalmente las variables independientes sino incorporar al modelo matemático actuarial más variables no consideradas hasta la fecha, hacerlo más representativo de la realidad, más representativo del sistema bajo estudio, incorporando principalmente las variables o factores referidos a la esperanza de vida individualizadas por edad y sexo así como las correspondientes rentas vitalicias temporales. Sin embargo, dada la variedad de casos que se pueden presentar en la práctica y que definirán a su vez diversos valores para las principales variables, en particular edades, sexos, tasas de interés técnico actuariales (TITA), cuentas individuales 
de capitalización (CIC), etc., entonces a fin de sensibilizar y darle la mayor consistencia posible al modelo propuesto, sí se hace también en tal sentido investigación experimental, ya que intencionalmente se hacen variar algunas de las más importantes variables independientes a manera de simulación en el modelo que representa lo que ocurre de manera más probable en la realidad.

En un principio se considerará la posibilidad de las rentas vitalicias en unidades monetarias constantes, trabajando con modelos matemáticos actuariales en unidades monetarias constantes, en nuevos soles reales, para luego incorporar el criterio del increasing o gradiente geométrica, que permitirá hacer crecer las pensiones no al ritmo inflacionario sino a una tasa de crecimiento diferente, debiéndose elaborar un modelo matemático actuarial en nuevos soles corrientes, tal como se aprecia en el diagrama de flujo siguiente, referido al diseño que interrelaciona las principales variables:

CIC, BR, i

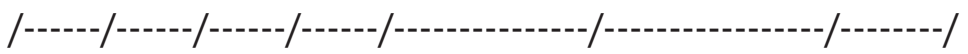

$\mathrm{x} \mathrm{x}+1 \mathrm{x}+2 \mathrm{x}+3 \mathrm{x}+4 \ldots \ldots \ldots \ldots \ldots \mathrm{x}+$ ESP VIDA............ $-1 \mathrm{w}$ (110 años)

$P \mathrm{P}(1+\mathrm{g}) \mathrm{P}(1+\mathrm{g})^{2} \mathrm{P}(1+\mathrm{g})^{3} \mathrm{P}(1+\mathrm{g})^{4} \ldots \ldots . \mathrm{P}(1+\mathrm{g})^{\text {ESP VIDA }} \ldots \ldots . \mathrm{P}(1+\mathrm{g})^{\mathrm{W}-1-\mathrm{X}}$

Donde:

$\mathrm{x}$, es la edad del afiliado y considerando su sexo

w, es la edad límite de la Tabla de Mortalidad vigente (110 años)

$\mathrm{P}$, es el monto de la pensión anual

$\mathrm{g}$, es la gradiente geométrica

i, es la Tasa de Interés Técnico Actuarial (TITA)

Es decir, primero se considerará lo existente en el sistema asegurador pensionario, que asume que el CIC más el BR deben alcanzar hasta los 110 años y luego el modelo matemático alternativo propuesto, que considera que el CIC y el BR deben alcanzar hasta la fecha más probable de fallecimiento del afiliado, la cual es definida por su correspondiente esperanza de vida destacando que: 
a. Las principales poblaciones bajo estudio son los afiliados a las AFPy los pensionistas del SPP diferenciados por sexo, edad, remuneraciones, $\mathrm{CIC}, \mathrm{BR}$, esperanzas de vida, etc., los que a abril del 2018 ascienden a 6'747,814 afiliados activos y 173,009 pensionistas

b. Los principales sujetos u objetos de análisis son: los afiliados a las AFP en actividad, los pensionistas del SPP y de las Compañías de Seguros, las AFP, las Compañías de Seguros Privadas, la Superintendencia de Bancos, Seguros y Administradoras de Fondos de Pensiones (SBS y AFP), la Oficina de Normalización Previsional (ONP), el Instituto Nacional de Estadística e Informática (INEI), etc.

\section{Resultados y discusión}

Se analizan, interpretan y discuten algunos de los principales resultados mediante los diferentes casos asociados a los modelos vigentes y propuestos, empezando por el tratamiento de las estimaciones de las esperanzas de vida necesarias para la elaboración de los modelos correspondientes.

\section{Caso Típico: Esperanzas de vida con la Tabla de Mortalidad de Beneficiarios Hombres (TM BH) vigente.}

Esta TM BH, se caracteriza porque en relación a la de RV H, tiene menores probabilidades de muerte (qx) en las primeras edades, pero a partir de los 26 dichas qx son mayores hasta los 86 años, siendo menores desde los 87 hasta los 99 y continúan siendo mayores desde los 100 hasta el fin de la tabla. Tal como se aprecia en el siguiente Cuadro № 02, las esperanzas de vida van disminuyendo en la medida que las probabilidades de muerte van aumentando con la edad.

Una persona de 50 años tiene una probabilidad de muerte de 0.00515 o 51.5 casos en diez mil, con una esperanza de vida de 29 años, es decir dicha persona podría fallecer a los 79 años. En cambio una persona de 70 años tiene una probabilidad de muerte de 0.02906 ó lo que es lo mismo 290.6 casos en diez mil, con una esperanza de vida de 14 años, es decir dicha persona podría fallecer a los 84 años. 
Se observa que las probabilidades (\%) de que un varón de edad actual $\mathrm{X}$ alcance los $110,100 \ldots . . . .65$ años van aumentando en la medida que va aumentando la edad. Es resaltante que una persona, aproximadamente entre 20 hasta los 60 años, tenga una probabilidad de llegar con vida hasta los 110 años de tan solo $0.0135 \%$ o lo que es lo mismo tan solo llegarían 1.35 personas de cada 10,000, es decir es sumamente difícil (más difícil que lo que se obtiene con la TM RV H, de 3 en diez mil).

También se aprecia que a partir de los 65 años, la mayoría de los fallecimientos, (más del 87\%, ocurrirá entre los 70 y 100 años).

Modelo Vigente en soles constantes de estimación de las rentas hasta los 110 años. Caso: Varones de 50 años y más con TM BH.

La ecuación de equilibrio actuarial fundamental nos dice que la CIC más el BR deben igualarse al valor presente actuarial o demográfico financiero de la renta vitalicia por cobrarse o lo que es lo mismo, el CIC más el BR deben servir para financiar el pago de la pensión hasta el fallecimiento del pensionista, que podría ocurrir a los 110 años, por lo que dicha ecuación es:

$$
\mathrm{CIC}+\mathrm{BR}=\mathrm{R}^{*} 12 * \mathrm{FASA}(1)
$$

Es decir, la CIC más el BR deben generar una pensión o renta R mensual hasta el límite de la tabla, hasta los 110 años, para lo cual se debe trabajar con un factor de actualización de flujos o series actuariales (FASA), referida al valor actual de una renta unitaria de vida entera inmediata de pago anticipado y cuya expresión en términos de los conmutadores actuariales aportados y empleados, entre otros, por Aníbal Allen, Arthur Anderson, Mario Coppini, José Gonzáles Galé, Antonio Lasheras, Hugo Palacios y Peter Thullen,citados en las referencias bibliográficas correspondientes, es: (Nx/Dx - 11/24), en donde Nx y Dx son los conmutadores actuariales que definen el FASA y 11/24 es un término de ajuste para flujos anuales anticipados con pagos " $m$ " veces al año, que proviene de $(m-1) / 2 m$, con $\mathrm{m}=12$.Por lo tanto:

$$
\mathrm{R}=(\mathrm{CIC}+\mathrm{BR}) /(12 *(\mathrm{Nx} / \mathrm{Dx}-11 / 24))(2)
$$

En este caso, tal como se aprecia en el Cuadro № 03A, se ha trabajado con una TITA del 3\% y con CIC más BR que van desde S/30,000.- hasta S/460,000.-, observándose que, en la medida que aumenta la edad, las 
RV también van aumentando debido a que el fondo hipotético de CIC más BR,en cuanto la persona se retira de mayor edad, tendrá menor esperanza de vida y dicho fondo hipotético constante para cada uno de los ocho casos, alcanzará para generar una pensión más alta pero que comprende menos años de vida esperados.

Por ejemplo, un varón de 65 años con un fondo de S/460,000.- podría cobrar S/2,253.48 en lugar de S/3,004.65, con una TITA del 3\% y unos gastos administrativos supuestos del 25\%.Tal como se aprecia en el Cuadro № 03B, se ha trabajado con una TITA del 4\% y con CIC más BR que van desde S/30,000.- hasta S/460,000.-, observándose nuevamente que, para todos los casos, en la medida que aumenta la edad, las RV también van aumentando. Otro caso es el de un varón de 65 años con un fondo de $S / 460,000$ que podría cobrar $S / 2,459.40$ en lugar de $S / 3,279.19$, con una TITA del $4 \%$ y unos gastos administrativos supuestos del 25\%. Estos montos de RV también son mayores que los obtenidos con la TM RVH, ya que esta TM BH contiene generalmente mayores probabilidades de muerte qx.

También es consistente la situación cuando la TITA disminuye a un 2\%, pues dicho varón de 65 años con un fondo de S/460,000.- podría cobrar S/2,052.83 en lugar de S/2,737.10, con dicha TITA del 2\% y unos gastos administrativos supuestos del $25 \%$. Es decir se verifica la relación directa que existe entre la RV y la TITA, pues al 4\% la RV era de $\mathrm{S} / 2,459.40$, al $3 \%$ de $\mathrm{S} / 2,253.48$ y al $2 \%$ de $\mathrm{S} / 2,052.83$, es decir a menor TITA menor RV (Cuadro $3 \mathrm{C}$ ). También es verificable que los montos de $\mathrm{RV}$ en dicho cuadro con TM BH son mayores que los que se obtendrían con la TM RVH.

\section{Modelo Propuesto en soles constantes de estimación de las rentas hasta la fecha de fallecimiento estimada por la Esperanza de Vida}

De manera similar a lo anteriormente expuesto y en contraste a lo vigente, se presentan los siguientes casos de estimación de las RV no hasta el final de las TM sino estimadas hasta las fechas de fallecimiento más probables, definidas por las esperanzas de vida (ex) de cada posible pensionista, según la edad, sexo y las tablas vigentes disponibles para los afiliados retirados. 


\section{Caso: Varones de 50 años y más con TM BH}

De manera análoga el CIC más el BR deben servir para financiar el pago de la pensión hasta el fallecimiento del pensionista, que ocurrirá de manera más probable no a los 110 años sino definida la fecha del fallecimiento por su esperanza de vida, tal como se especifica en el Cuadro № 02 trabajado precisamente con la TM BH, por lo que dicha ecuación para este caso es:

$$
\mathrm{CIC}+\mathrm{BR}=\mathrm{R}^{*} 12 * \text { FASAT }(3)
$$

Es decir, la CIC más el BR deben generar una pensión o renta R mensual hasta la fecha de fallecimiento más probable definida por la ex, para lo cual se debe trabajar con un factor de actualización de flujos o series actuariales temporales (FASAT), semejante a los factores de ingeniería económica empleados por, entre otros, Gabriel Baca, Anthony Tarquin y George Taylor, citados en la bibliografía, estando el FASAT referido al valor actual de una renta unitaria anual temporal inmediata de pago anticipado y cuya expresión en términos de los conmutadores actuariales también referidos en la bibliografía correspondiente es: $(\mathrm{Nx}-\mathrm{Nx}+\mathrm{ex}) /$ Dx, la cual cuando es pagada " $m$ " veces al año se ajusta con el siguiente término $((m-1) / 2 m) *(1-e x E x)$, con exEx referido a un dotal puro o capital diferido, expresado por exEx $=\mathrm{Dx}+\mathrm{ex} / \mathrm{Dx}$, de donde se obtiene al igual que antes:

$(N x-N x+e x) / D x-((m-1) / 2 m) *(1-e x E x)=$

$\left(\mathrm{Nx}-\mathrm{Nx}+\mathrm{ex}-11 / 24^{*}(\mathrm{Dx}-\mathrm{Dx}+\mathrm{ex})\right) / \mathrm{Dx}$

Y por lo tanto: $\mathrm{R}=((\mathrm{CIC}+\mathrm{BR}) * \mathrm{Dx})) /(12 *((\mathrm{Nx}-\mathrm{Nx}+\mathrm{ex}-11 / 24 *(\mathrm{Dx}-$ Dx+ex))) (4)

En este caso, tal como se aprecia en el Cuadro № 04A, se ha trabajado con una TITA del 3\% y con CIC más BR que van desde S/30,000.- hasta S/460,000.-, observándose que, para todos los casos, en la medida que aumenta la edad, las RV también van aumentando.

Un varón de 65 años con un fondo de S/460,000.- podría cobrar $\mathrm{S} / 2,678.95$ en lugar de $\mathrm{S} / 3,571.93$, con una TITA del $3 \%$ y unos gastos administrativos supuestos del 25\%. Si comparamos estos montos con relación al Cuadro № 03A en el que se trabajó con la TM BH también 
al 3\%, pero con vida entera hasta los 110 años, se pueden apreciar las mayores RV propuestas. Con la ex el varón de 65 años cobraría $S / 2,678.95$, en cambio con vida entera hasta los 110 años solo S/2,253.48 (casi 19\% más).Tal como se aprecia en el Cuadro № 04B, se ha trabajado con una TITA del 4\% y con CIC más BR que va desde S/30,000.- hasta S/460,000., observándose nuevamente que, para todos los casos, en la medida que aumenta la edad, las RV también van aumentando. Un varón de 65 años con un fondo de $\mathrm{S} / 460,000$.- podría cobrar $\mathrm{S} / 2,860.13$ en lugar de $\mathrm{S} / 3,813.50$, con una TITA del $4 \%$ y unos gastos administrativos supuestos del $25 \%$. Igualmente, con la ex el varón de 65 años cobraría $S / 2,860.13$, en cambio con vida entera hasta los 110 años solo S/2,459.40 (16\% más).

Otro caso comprobable es cuando la TITA disminuye a un 2\%; dicho varón de 65 años con un fondo de S/460,000.- podría cobrar S/2,502.40 en lugar de $\mathrm{S} / 3,336.54$, con dicha TITA del $2 \%$ y unos gastos administrativos supuestos del 25\% (Cuadro № 04C). Igualmente, con la ex el varón de 65 años cobraría $S / 2,502.40$, en cambio con vida entera hasta los 110 años solo $\mathrm{S} / 2,052.83$ (casi $22 \%$ más).

Es decir se verifica no solo la relación directa que existe entre la RV y la TITA, pues al $4 \%$ la RV era de $S / 2,860.13$ (mayor que $S / 2,459.40$ ), al $3 \%$ de $\mathrm{S} / 2,678.95$ (mayor que $\mathrm{S} / 2,253.48$ ) y al $2 \%$ de $\mathrm{S} / 2,502.40$ (mayor que $S / 2,052.83$ ), sino que también se comprueba en todos los casos que con la fecha de fallecimiento estimada por la ex y el FASAT se cobra mayor $\mathrm{RV}$ que con el criterio vigente del fallecimiento hasta los 110 años, de los Cuadros 3 A, B y C.

Se podrían incluir otros temas asociados a otras tablas actuariales, con diferentes edades y para ambos sexos, con estimaciones de esperanzas de vida diferenciadas u otros modelos propuestos en soles constantes o en soles corrientes u opciones de pensionistas con cónyuges menores o mayores que el beneficiario, o casos con gradientes, o comparaciones y evaluaciones entre modelos en soles constantes y soles corrientes con gradiente, etc., sin embargo mayor referencia detallada de ello y otros conceptos podrán revisarse en la mencionada tesis "Modelos matemáticos actuariales y fondos previsionales pensionarios: rentas vitalicias hasta 110 años o hasta cuando el pensionista fallezca de manera más probable" documento que es base de este artículo. 
Pensamiento Crítico Vol. 23. N 1

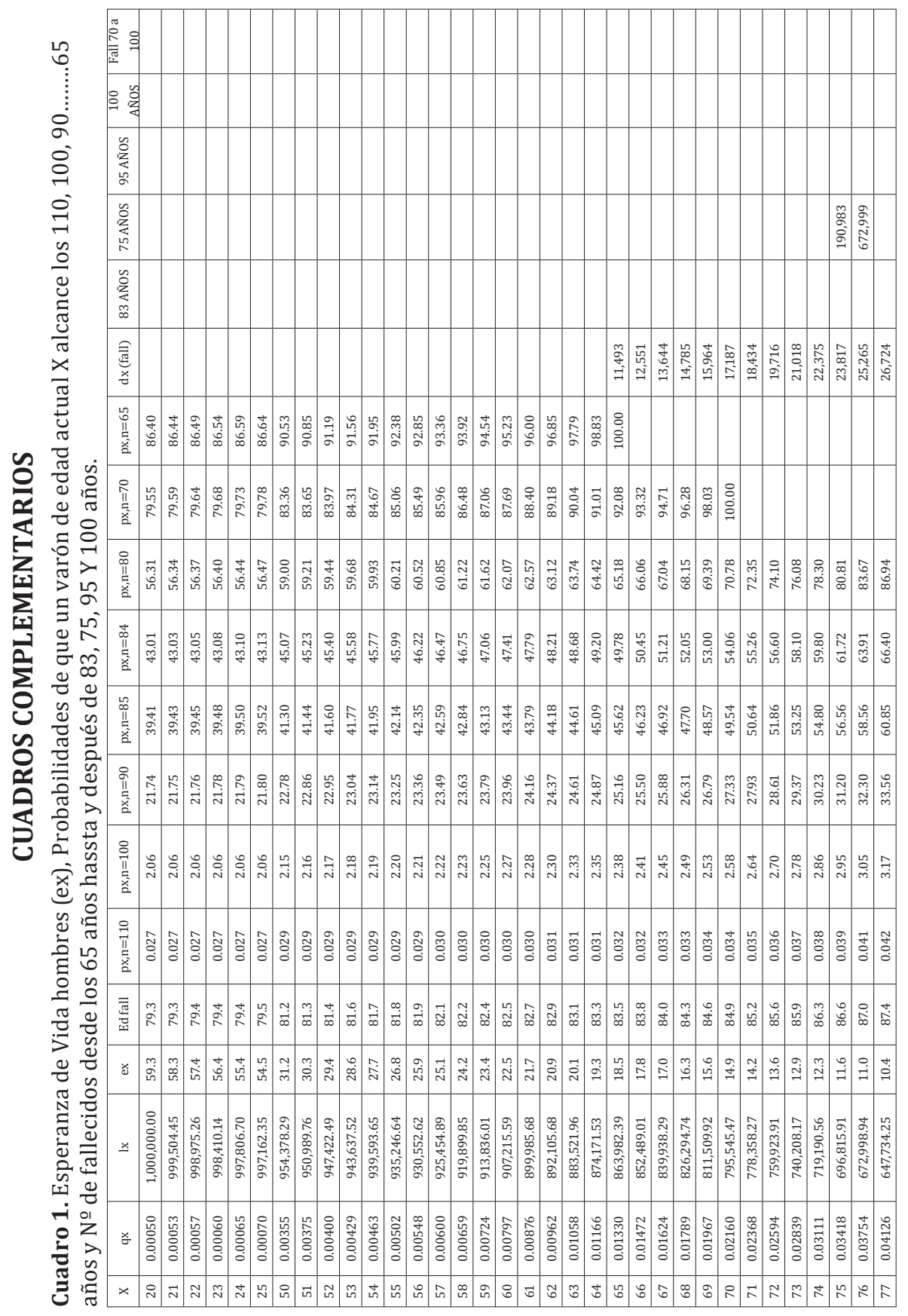




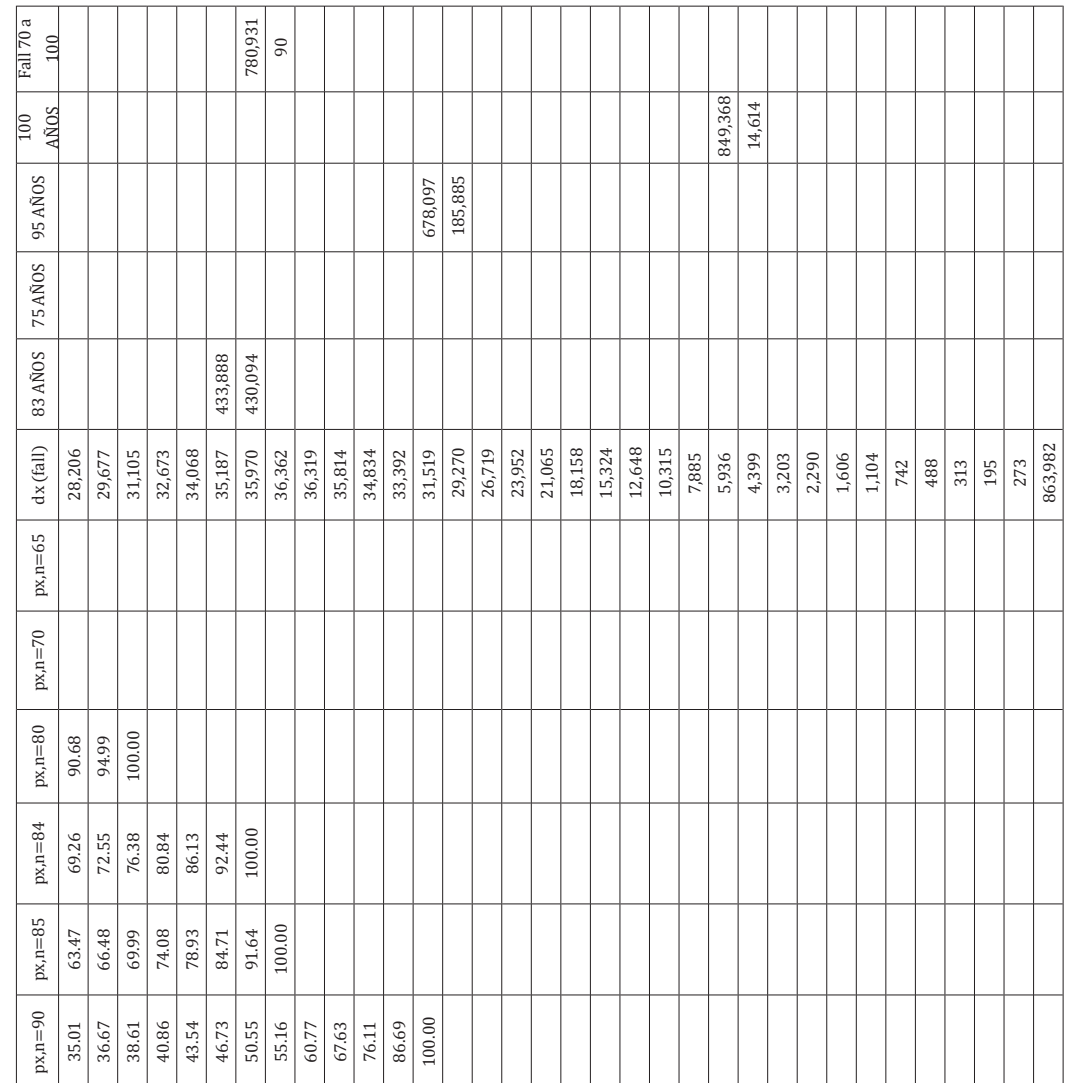

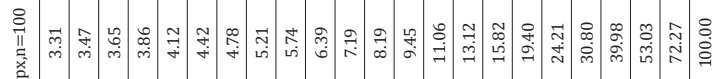

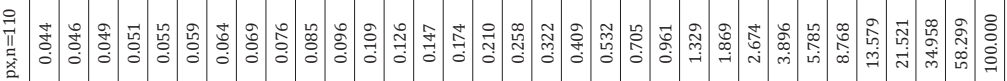

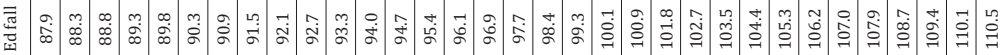

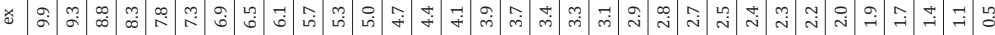

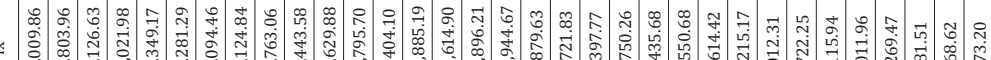

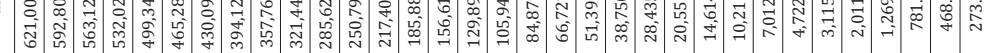

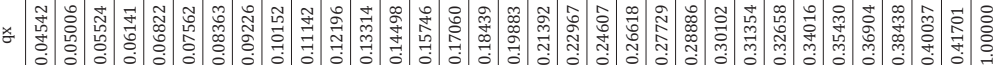

メ

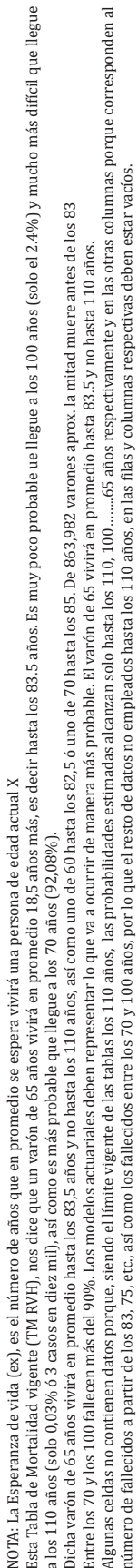


Pensamiento Crítico Vol. 23. № 1

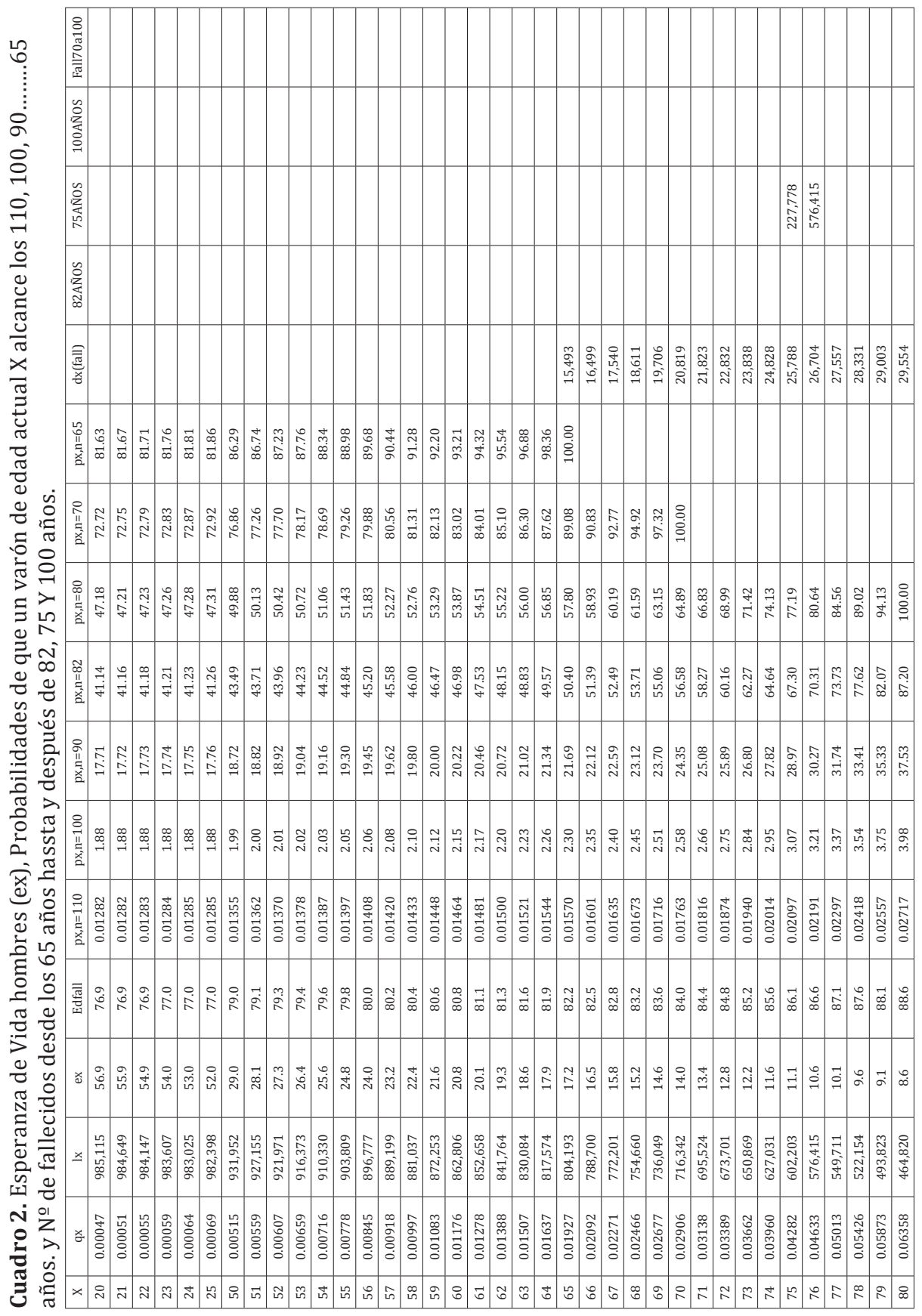


José E. Cavero Vicentelo, Miguel A. Pinglo Ramírez

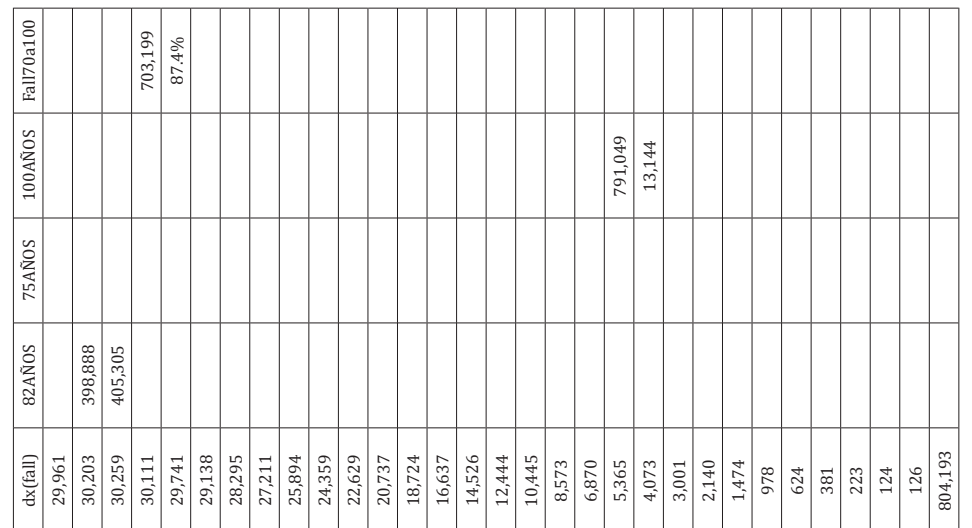

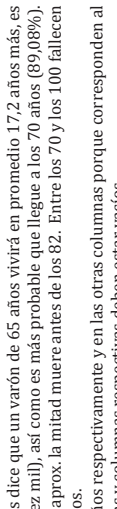

는

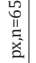

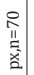

离

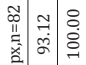

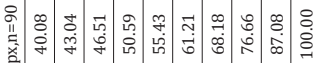

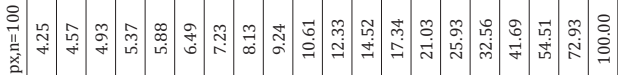

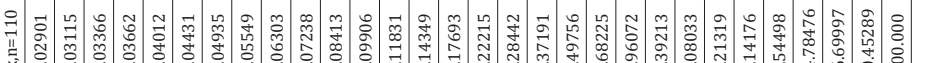

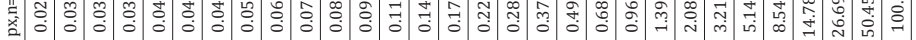

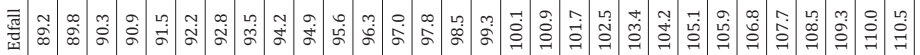

๔

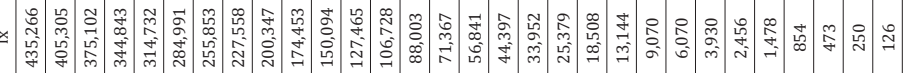

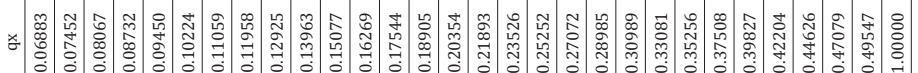

メ

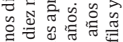

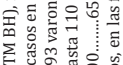

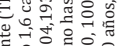

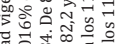

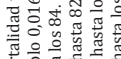

递

造造卷

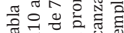

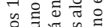

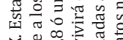

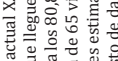

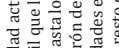

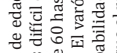

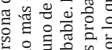

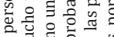

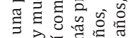

뚱ำ

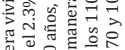

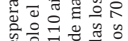

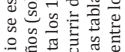

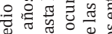
봉응

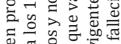

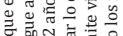

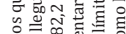

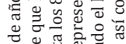

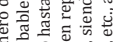

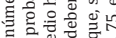
๘

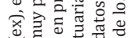

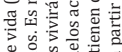

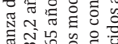

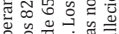

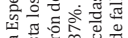

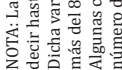


Pensamiento Crítico Vol. 23. № 1

\begin{tabular}{|c|c|c|c|c|c|c|c|c|c|c|c|c|c|c|c|c|c|c|c|c|c|c|c|c|c|c|c|c|c|c|c|c|c|}
\hline & $\begin{array}{l}0 \\
0 \\
0 \\
0 \\
0 \\
0 \\
0 \\
\vdots \\
\end{array}$ & $\begin{array}{l}0 \\
0 \\
0 \\
0 \\
0 \\
0 \\
0\end{array}$ & 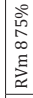 & & & & 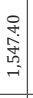 & $\begin{array}{ll}\vec{a} \\
\infty \\
\vdots \\
i n\end{array}$ & $\begin{array}{l}a \\
0 \\
0 \\
0\end{array}$ & 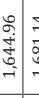 & 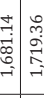 & 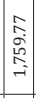 & 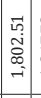 & 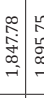 & 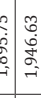 & 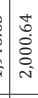 & & & 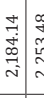 & 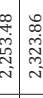 & & & 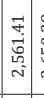 & 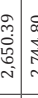 & 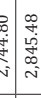 & & 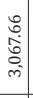 & 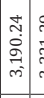 & & & & & \\
\hline & $\begin{array}{r}0 \\
0 \\
0 \\
0 \\
0 \\
0 \\
0\end{array}$ & & & & & & & 它 & J & 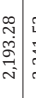 & 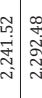 & & 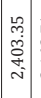 & 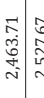 & $\begin{array}{lll}0 \\
\end{array}$ & 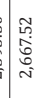 & $\mid$ & 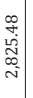 & 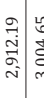 & 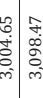 & & $\begin{array}{l}\hat{m} \\
\tilde{j} \\
0 \\
\tilde{m}\end{array}$ & 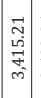 & 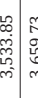 & 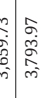 & a. & 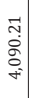 & 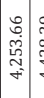 & 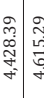 & & & & \\
\hline & $\begin{aligned} & 0 \\
& 0 \\
& 0 \\
& 0 \\
& 0 \\
& 0 \\
& 0 \\
& 0\end{aligned}$ & & & & & & & 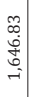 & : & | & 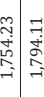 & 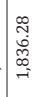 & $\begin{array}{c}\infty \\
\infty \\
0 \\
\infty \\
\oplus \\
\rightarrow\end{array}$ & 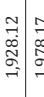 & 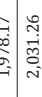 & 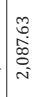 & 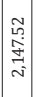 & 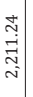 & 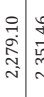 & 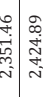 & & 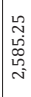 & 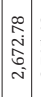 & 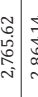 & 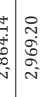 & $\begin{array}{c}\tilde{m} \\
\overrightarrow{0} \\
\mathbb{g} \\
\end{array}$ & 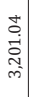 & 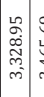 & 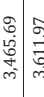 & & 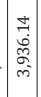 & $\bar{F}$ & \\
\hline & $\begin{array}{l}0 \\
0 \\
0 \\
0 \\
0 \\
0 \\
0\end{array}$ & $\left|\begin{array}{l}0 \\
\vdots \\
\vdots \\
\dot{q}\end{array}\right|$ & & & & & & 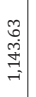 & 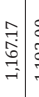 & 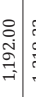 & 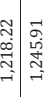 & 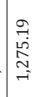 & $\begin{array}{l}7 \\
\stackrel{0}{0} \\
m \\
\rightarrow \\
\rightarrow\end{array}$ & 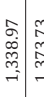 & 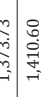 & 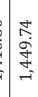 & 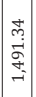 & 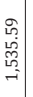 & 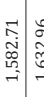 & 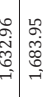 & & 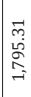 & $\left|\begin{array}{l}0 \\
0 \\
0 \\
0 \\
0 \\
0 \\
-1\end{array}\right|$ & 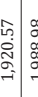 & 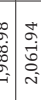 & $\left|\begin{array}{l}\infty \\
\infty \\
0 \\
0 \\
\sim \\
\sim\end{array}\right|$ & $\mid$ & 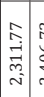 & 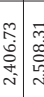 & & 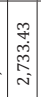 & $\infty$ & \\
\hline & $\begin{array}{r}0 \\
\text { + } \\
\text { + } \\
0 \\
0 \\
0 \\
0\end{array}$ & & & & & & & & & & 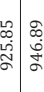 & $\begin{array}{l}2 \\
8 \\
0\end{array}$ & $\begin{array}{l}\sigma \\
\stackrel{\sigma}{0} \\
\dot{\alpha} \\
\sigma\end{array}$ & 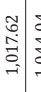 & 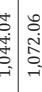 & 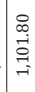 & 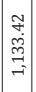 & فئّ & 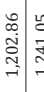 & 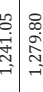 & 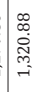 & $\begin{array}{l}F \\
\dot{H} \\
\dot{0} \\
\rightarrow \\
\rightarrow\end{array}$ & 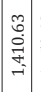 & 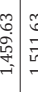 & 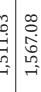 & 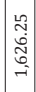 & 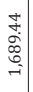 & & 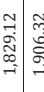 & 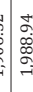 & 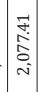 & $\overrightarrow{\mathrm{N}}$ & \\
\hline & $\begin{array}{l}0 \\
0 \\
0 \\
0 \\
0 \\
1 \\
10\end{array}$ & & & & & & & & 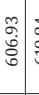 & 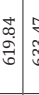 & & $\begin{array}{l}\text { के } \\
\text { : }\end{array}$ & $\mid \begin{array}{l}3 \\
\vdots \\
0 \\
0\end{array}$ & 党 & 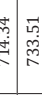 & 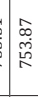 & 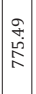 & 号 & 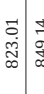 & 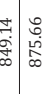 & & 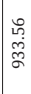 & 경 & 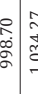 & 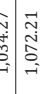 & $\mathbb{\exists}$ & 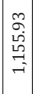 & 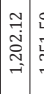 & 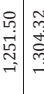 & 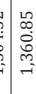 & 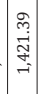 & 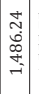 & \\
\hline & 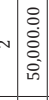 & & & & & & & 足 & 令 & 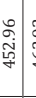 & 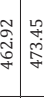 & 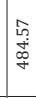 & $\mid \begin{array}{l}\vec{m} \\
0 \\
o \\
\sigma\end{array}$ & 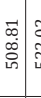 & 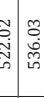 & $\begin{array}{l}8 \\
0 \\
0 \\
h \\
h\end{array}$ & 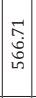 & 岕 & 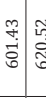 & 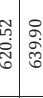 & : & 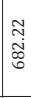 & 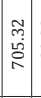 & 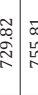 & 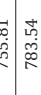 & $\begin{array}{l}m \\
\tilde{m} \\
\vec{\infty}\end{array}$ & 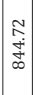 & & 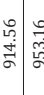 & 尔 & & 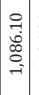 & \\
\hline & $\begin{array}{r}0 \\
0 \\
0 \\
0 \\
0 \\
0 \\
0\end{array}$ & & & & & & & 勇 & 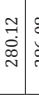 & 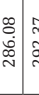 & 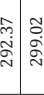 & 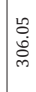 & $\mid \begin{array}{c}\infty \\
\stackrel{\infty}{m} \\
\tilde{m}\end{array}$ & 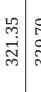 & 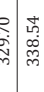 & 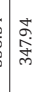 & 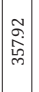 & 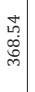 & 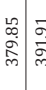 & 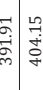 & $\frac{7}{7}$ & \begin{tabular}{|l}
$\infty$ \\
$\infty$ \\
$\dot{p}$ \\
$\dot{q}$
\end{tabular} & 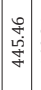 & 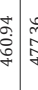 & 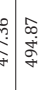 & 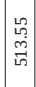 & 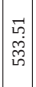 & 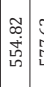 & 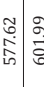 & & 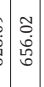 & 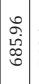 & \\
\hline & 8 & $\frac{\mathrm{m}}{\mathrm{m}}$ & & & & & & ָㅗㅀ & 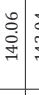 & 荬 & & $\stackrel{\sim}{\sim}$ & 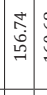 & لو: & 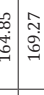 & 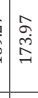 & 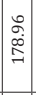 & 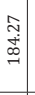 & 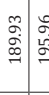 & 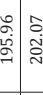 & & $\begin{array}{l}\text { J } \\
\text { 离 }\end{array}$ & $\stackrel{\substack{\sim \\
\sim}}{\sim}$ & 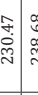 & 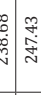 & & 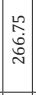 & & 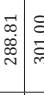 & s: & & $\mid$\begin{tabular}{l}
$\infty$ \\
\multirow{2}{*}{} \\
\end{tabular} & \\
\hline & & & & & 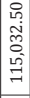 & 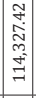 & 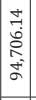 & 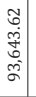 & & & 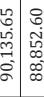 & & 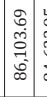 & 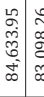 & 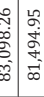 & 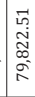 & 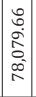 & 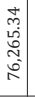 & 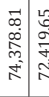 & 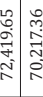 & 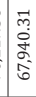 & 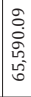 & $\begin{array}{c}0 \\
0 \\
0 \\
\tilde{0} \\
\tilde{0}\end{array}$ & 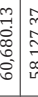 & 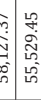 & & 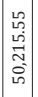 & 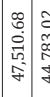 & 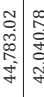 & 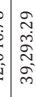 & 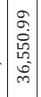 & $\begin{array}{l}0 \\
0 \\
\vdots \\
\infty \\
\tilde{m} \\
\tilde{m}\end{array}$ & \\
\hline & & & & & & \begin{tabular}{|c|} 
\\
0 \\
+ \\
$\dot{p}$ \\
$m$
\end{tabular} & 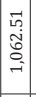 & 倠 & & 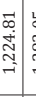 & تَ & $\begin{array}{l}\infty \\
0 \\
u \\
0 \\
0\end{array}$ & 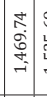 & 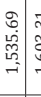 & 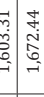 & 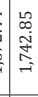 & 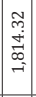 & 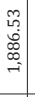 & 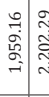 & 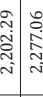 & 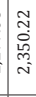 & 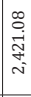 & 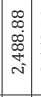 & 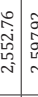 & 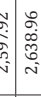 & 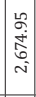 & 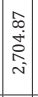 & 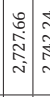 & 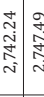 & : & 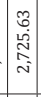 & : & \\
\hline & & & & & 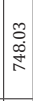 & 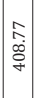 & 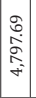 & & & 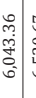 & 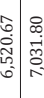 & 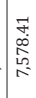 & 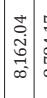 & 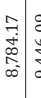 & 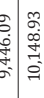 & 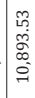 & $\mid \begin{array}{l}\vec{f} \\
\dot{0} \\
0 \\
\vec{\exists} \\
\vec{\exists}\end{array}$ & 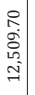 & 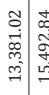 & 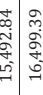 & 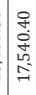 & 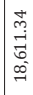 & 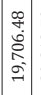 & 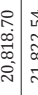 & 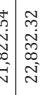 & \begin{tabular}{|c|c}
$\infty$ \\
0 \\
0 \\
$\infty$ \\
$\sim$ \\
$\sim$ \\
$\tilde{n}$
\end{tabular} & 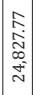 & 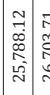 & 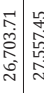 & 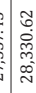 & 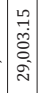 & 类 & \\
\hline & & & $\ddot{x}$ & 6 & 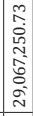 & 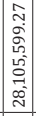 & 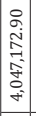 & 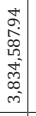 & 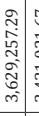 & 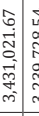 & 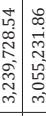 & 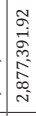 & 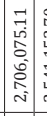 & 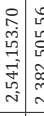 & 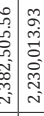 & 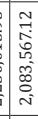 & 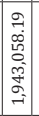 & 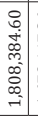 & 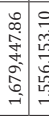 & 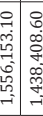 & 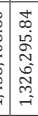 & 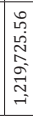 & 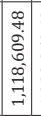 & 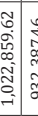 & 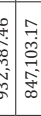 & \begin{tabular}{|l|} 
\\
\\
0 \\
0 \\
0 \\
$b$ \\
0 \\
0
\end{tabular} & $\mid \begin{array}{l}\mid \\
0 \\
0 \\
0 \\
0 \\
0 \\
0 \\
0 \\
0\end{array}$ & 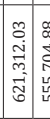 & 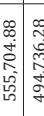 & 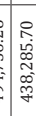 & 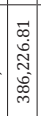 & 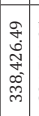 & \\
\hline & å & & 宭 & 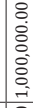 & & 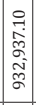 & & ง & 离 & 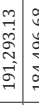 & 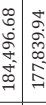 & E & 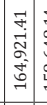 & 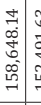 & 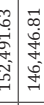 & 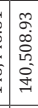 & 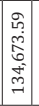 & 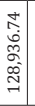 & 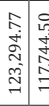 & 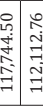 & & 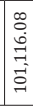 & 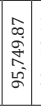 & 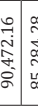 & 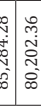 & 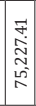 & 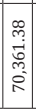 & 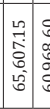 & 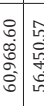 & 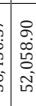 & 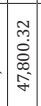 & 岁 & \\
\hline & & & $\underline{\simeq}$ & & & & & $\stackrel{8}{2}$ & & 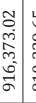 & 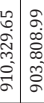 & ڤ్ & \begin{tabular}{c|c}
$\infty$ \\
$\infty$ \\
$\infty$ \\
0 \\
0 \\
0 \\
$\infty$ \\
$\infty$
\end{tabular} & 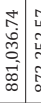 & 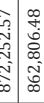 & 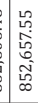 & 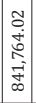 & $\begin{array}{l}\vec{\sigma} \\
0 \\
0 \\
0 \\
0 \\
\infty \\
\infty\end{array}$ & 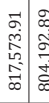 & 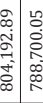 & : & 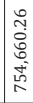 & 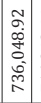 & 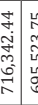 & 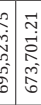 & \begin{tabular}{|c|c}
0 \\
$\infty$ \\
0 \\
0 \\
$\infty$ \\
0 \\
$b$ \\
$b$
\end{tabular} & 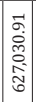 & 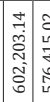 & 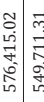 & 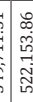 & $\begin{array}{c}\vec{u} \\
\tilde{z} \\
w \\
\tilde{g} \\
\tilde{g}\end{array}$ & 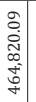 & \\
\hline & & & $\hat{\sigma}$ & 10 & & & 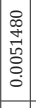 & $\stackrel{\circ}{\circ}$ & & 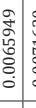 & 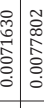 & 0 & 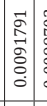 & 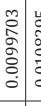 & 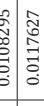 & $0_{0}^{\circ}$ & $\mid$\begin{tabular}{|c}
$\overrightarrow{0}$ \\
$\infty$ \\
0 \\
0 \\
0 \\
0 \\
0
\end{tabular} & $\begin{array}{ll} \\
0 \\
0 \\
0 \\
\vdots \\
0 \\
0 \\
0\end{array}$ & 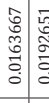 & 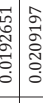 & & 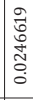 & 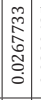 & 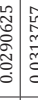 & 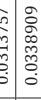 & $\begin{array}{l}\mid \\
0 \\
0 \\
0 \\
0\end{array}$ & $\mid$\begin{tabular}{|c}
0 \\
0 \\
0 \\
$\alpha$ \\
0 \\
0 \\
0 \\
0
\end{tabular} & 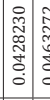 & 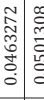 & 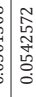 & $\begin{array}{l}\infty \\
0 \\
0 \\
0 \\
0 \\
0 \\
\dot{0}\end{array}$ & $\circ$ & \\
\hline & & & $\star$ & & & $\sim$ & & हो & & î & 苋织 & 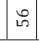 & in & $\begin{array}{c}\infty \\
\llcorner\end{array}$ & 68 & 5 & ก & 8 & की & L. & & $\infty$ & o & & $\therefore \approx$ & & ‡ & 25 & $\therefore R$ & & 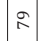 & & \\
\hline
\end{tabular}


José E. Cavero Vicentelo, Miguel A. Pinglo Ramírez
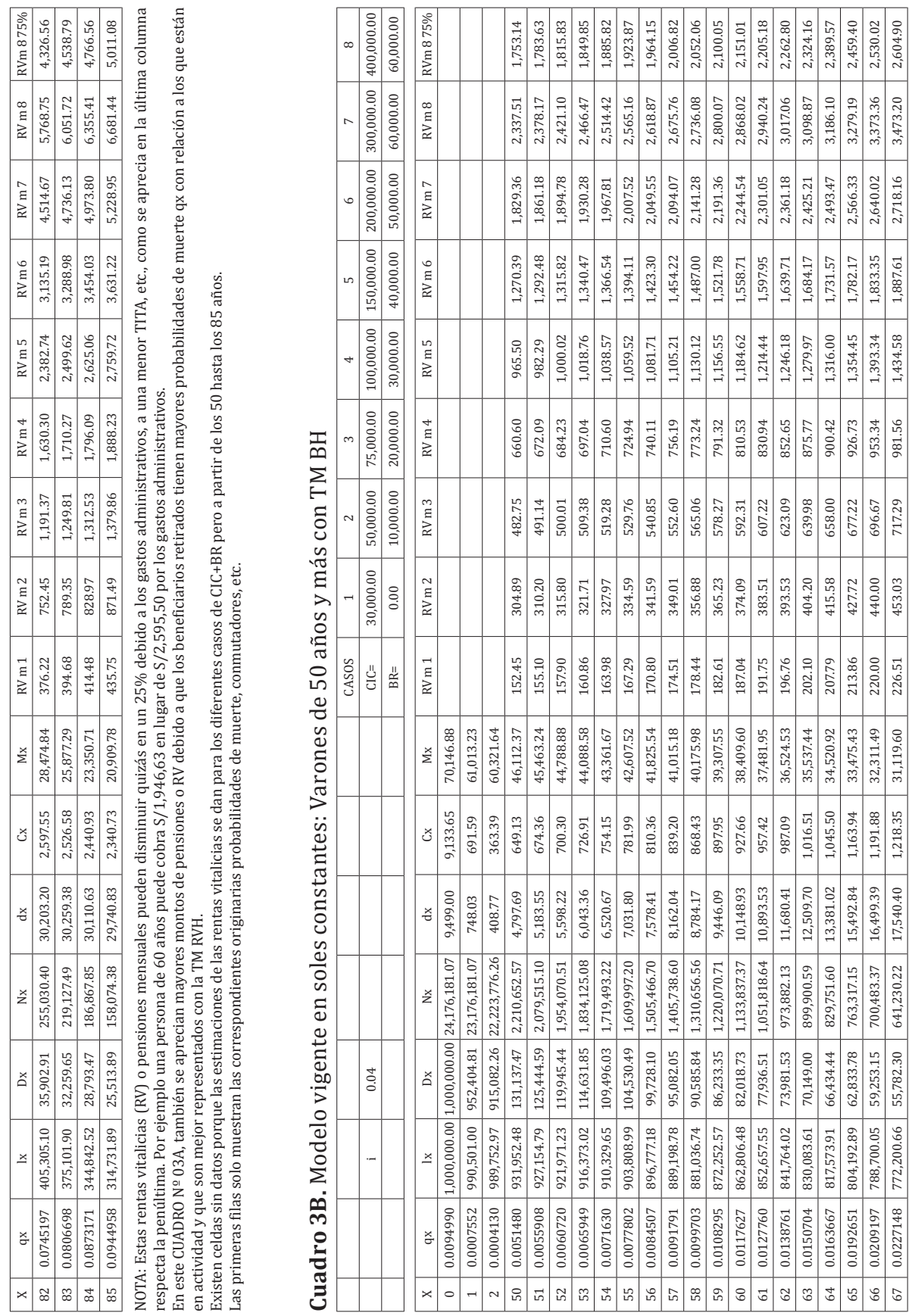
Pensamiento Crítico Vol. 23. № 1

\begin{tabular}{|c|c|c|c|c|c|c|c|c|c|c|c|c|c|c|c|c|c|c|}
\hline 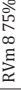 & $\begin{array}{l}\vec{m} \\
\vec{m} \\
\stackrel{5}{0} \\
\stackrel{N}{N}\end{array}$ & 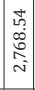 & 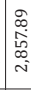 & 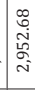 & $\begin{array}{l}\vec{\infty} \\
\tilde{m} \\
\overrightarrow{0} \\
\vec{c} \\
\dot{m}\end{array}$ & 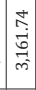 & 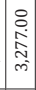 & $\begin{array}{l}0 \\
0 \\
\vdots \\
0 \\
\vdots \\
\dot{1} \\
m\end{array}$ & 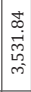 & 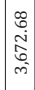 & $\mid \begin{array}{c}\vec{F} \\
\tilde{n} \\
\infty \\
\tilde{m} \\
\dot{m}\end{array}$ & \begin{tabular}{|c|}
$\vec{\infty}$ \\
$\vec{\Phi}$ \\
$\stackrel{0}{0}$ \\
ले
\end{tabular} & 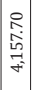 & 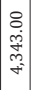 & 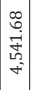 & 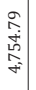 & & $\begin{array}{l}\infty \\
\infty \\
\infty \\
\infty \\
\text { N̦ } \\
\text { in }\end{array}$ \\
\hline$\stackrel{\infty}{{ }^{\infty}}$ & $\begin{array}{l}\infty \\
0 \\
\sigma \\
\sigma \\
2 \\
m \\
m\end{array}$ & 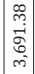 & $\begin{array}{l}\tilde{N} \\
\text { Th } \\
0 \\
0 \\
0 \\
\text { m. }\end{array}$ & $\begin{array}{l}\tilde{\sigma} \\
\delta \\
\tilde{\sigma} \\
\text { m. }\end{array}$ & 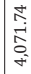 & 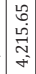 & 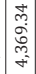 & 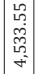 & $\begin{array}{l}\exists \\
\vec{a} \\
\stackrel{2}{2} \\
+\end{array}$ & $\begin{array}{l}\vec{\sigma} \\
\dot{\alpha} \\
\alpha \\
0 \\
+\end{array}$ & 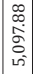 & 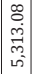 & 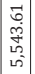 & $\mid \begin{array}{c}\hat{0} \\
0 \\
2 \\
\hat{L} \\
L^{2}\end{array}$ & $\left|\begin{array}{l}\infty \\
0 \\
h \\
น \\
0 \\
0 \\
0 \\
0\end{array}\right|$ & $\left|\begin{array}{c}\tilde{N} \\
\hat{D} \\
\tilde{m} \\
\text { b }\end{array}\right|$ & & $\begin{array}{l}\text { से } \\
\text { तू } \\
\text { S. }\end{array}$ \\
\hline$\stackrel{\Xi}{\sharp}$ & 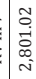 & $\mid \begin{array}{l}\vec{\alpha} \\
\infty \\
\infty \\
\infty \\
i\end{array}$ & 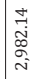 & $\begin{array}{c}0 \\
0 \\
\dot{0} \\
0 \\
0 \\
\text { m. }\end{array}$ & 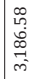 & $\begin{array}{l}\vec{N} \\
\dot{\alpha} \\
\tilde{N} \\
\tilde{m}\end{array}$ & 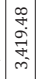 & $\begin{array}{c}0 \\
0 \\
o \\
o \\
\omega \\
\omega \\
m \\
m\end{array}$ & $\begin{array}{l}0 \\
\tilde{m} \\
\omega \\
0 \\
0 \\
\tilde{m}\end{array}$ & $\begin{array}{l}0 \\
\tilde{n} \\
\tilde{D} \\
\infty \\
\tilde{m}\end{array}$ & \begin{tabular}{|c}
0 \\
0 \\
0 \\
0 \\
$o$ \\
m.
\end{tabular} & 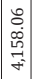 & $\begin{array}{c}\stackrel{f}{f} \\
\infty \\
m \\
m \\
\stackrel{\sigma}{*}\end{array}$ & 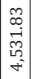 & 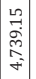 & 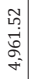 & & 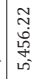 \\
\hline$\vec{a}$ & 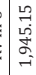 & $\mid \begin{array}{c}\infty \\
\vec{b} \\
\vdots \\
\vdots \\
i\end{array}$ & $\begin{array}{l}\text { Oे } \\
\hat{2} \\
\hat{0} \\
\text { i. }\end{array}$ & 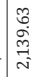 & 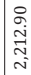 & 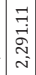 & 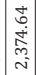 & 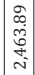 & 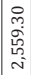 & 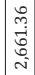 & 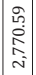 & 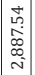 & $\begin{array}{c}0 \\
0 \\
\stackrel{j}{0} \\
0 \\
m\end{array}$ & 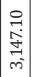 & 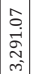 & 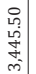 & & $\begin{array}{l}\tilde{D} \\
\dot{0} \\
\stackrel{0}{0} \\
\dot{0}\end{array}$ \\
\hline & 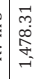 & 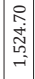 & $\begin{array}{l}\sigma \\
\sigma \\
\alpha \\
\alpha \\
\alpha \\
-1\end{array}$ & 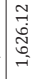 & $\begin{array}{l}\vec{\infty} \\
\overrightarrow{0} \\
\overrightarrow{0} \\
- \\
-1\end{array}$ & $\begin{array}{c}\stackrel{2}{T} \\
\vec{T} \\
\stackrel{+}{-}\end{array}$ & $\begin{array}{l}m \\
\stackrel{f}{+} \\
\dot{+} \\
\stackrel{\alpha}{-}\end{array}$ & 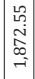 & 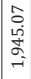 & 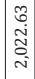 & 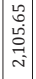 & 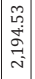 & 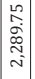 & 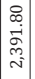 & 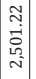 & 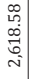 & & 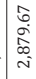 \\
\hline$\vec{x}$ & & 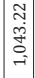 & $\begin{array}{l}\infty \\
\infty \\
0 \\
0 \\
0 \\
0 \\
-1\end{array}$ & 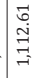 & $\begin{array}{l}\text { E } \\
\text { D. } \\
\text { in } \\
\text { - }\end{array}$ & 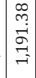 & $\mid \begin{array}{c}\vec{\infty} \\
\dot{+} \\
\overrightarrow{\tilde{n}} \\
\overrightarrow{-}\end{array}$ & 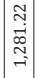 & 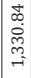 & $\begin{array}{l}\vec{\sigma} \\
\dot{\omega} \\
0 \\
\tilde{m} \\
\overrightarrow{-}\end{array}$ & 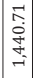 & 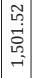 & 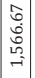 & 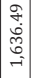 & $\mid \begin{array}{c}0 \\
\stackrel{2}{7} \\
\vec{i} \\
=\end{array}$ & $\mid \begin{array}{c}0 \\
0 \\
\dot{2} \\
\\
=\end{array}$ & & 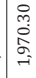 \\
\hline$\stackrel{2}{*}$ & $\begin{array}{l}\stackrel{0}{2} \\
\text { مू. }\end{array}$ & 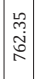 & 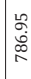 & $\begin{array}{l}\stackrel{\bullet}{\circ} \\
\stackrel{\infty}{\infty}\end{array}$ & 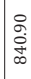 & $\begin{array}{l}\tilde{S} \\
0 \\
0 \\
\infty \\
\infty\end{array}$ & $\begin{array}{l}0 \\
\tilde{~} \\
\tilde{~} \\
\sigma\end{array}$ & 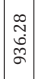 & 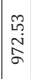 & $\begin{array}{c}\tilde{N} \\
\vec{G} \\
\overrightarrow{-} \\
-\overrightarrow{-}\end{array}$ & 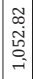 & $\mid \begin{array}{c}\hat{N} \\
\hat{N} \\
\hat{o} \\
-i\end{array}$ & $\mid$\begin{tabular}{c}
$\infty$ \\
$\infty$ \\
$\stackrel{+}{+}$ \\
\multirow{-}{-}{} \\
-1
\end{tabular} & 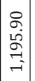 & 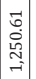 & 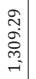 & & 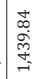 \\
\hline $\mathbb{\Xi}$ & 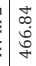 & \begin{tabular}{|c|}
$\infty$ \\
$\stackrel{\infty}{+}$ \\
$\stackrel{+}{+}$ \\
$\stackrel{+}{+}$
\end{tabular} & 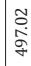 & 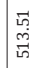 & $\begin{array}{l}0 \\
\text { in } \\
\text { in }\end{array}$ & $\begin{array}{l}\hat{\alpha} \\
\infty \\
\dot{\sigma} \\
\dot{H} \\
\end{array}$ & $\begin{array}{l}\vec{\sigma} \\
\hat{\sigma} \\
\llcorner\end{array}$ & 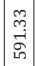 & $\begin{array}{l}\tilde{N} \\
\stackrel{+}{\vec{b}}\end{array}$ & $\begin{array}{l}m \\
\infty \\
\tilde{\sigma}\end{array}$ & 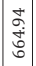 & $\begin{array}{l}\vec{D} \\
\dot{\rho} \\
\sigma \\
\sigma\end{array}$ & $\begin{array}{l}\infty \\
\stackrel{\infty}{ } \\
\stackrel{\sim}{N} \\
\end{array}$ & 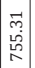 & $\left|\begin{array}{l}\infty \\
\infty \\
\vdots \\
\infty \\
\vdots\end{array}\right|$ & 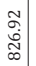 & & $\begin{array}{l}\hat{m} \\
\text { S̆ }\end{array}$ \\
\hline 1 & $\begin{array}{l}\text { Fै } \\
\text { लू } \\
\text { N }\end{array}$ & 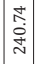 & $\begin{array}{l}\overrightarrow{5} \\
\infty \\
\stackrel{0}{N}\end{array}$ & 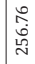 & 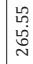 & \begin{tabular}{l}
$\Re$ \\
\multirow{2}{*}{} \\
$\stackrel{+}{*}$
\end{tabular} & 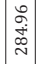 & 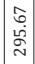 & $\begin{array}{l}\mathcal{N} \\
\overrightarrow{\tilde{D}} \\
\tilde{\tilde{m}}\end{array}$ & $\begin{array}{l}0 \\
\stackrel{2}{g} \\
\text { mे }\end{array}$ & 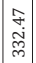 & 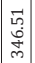 & 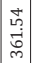 & 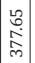 & 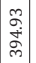 & $\mid \begin{array}{l}0 \\
+ \\
m \\
\exists \\
\xi\end{array}$ & & 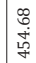 \\
\hline$\sum_{\Sigma}^{\infty}$ & 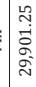 & 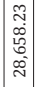 & 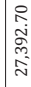 & 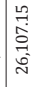 & 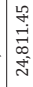 & 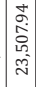 & 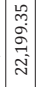 & $\begin{array}{l}L \\
\infty \\
\infty \\
\infty \\
\infty \\
\infty \\
0 \\
0\end{array}$ & 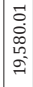 & 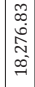 & 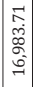 & 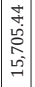 & 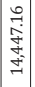 & 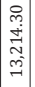 & 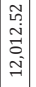 & 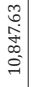 & & 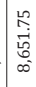 \\
\hline 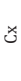 & 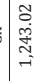 & 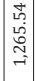 & 荿 & 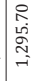 & 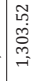 & $\begin{array}{c}\mathfrak{r} \\
0 \\
0 \\
0 \\
-7 \\
-7\end{array}$ & $\begin{array}{l}0 \\
1 \\
0 \\
-3 \\
-7 \\
-1\end{array}$ & 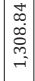 & $\begin{array}{l}\infty \\
\dot{m} \\
\stackrel{0}{0} \\
\vec{\sim}\end{array}$ & $\begin{array}{l}\mathcal{N} \\
\mathcal{M} \\
\tilde{N} \\
\sim \\
\sim\end{array}$ & 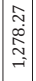 & 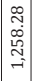 & $\begin{array}{l}0 \\
\infty \\
\tilde{N} \\
\stackrel{-}{-} \\
-\end{array}$ & 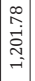 & 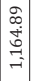 & 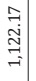 & & $\begin{array}{l}m \\
\hat{\sigma} \\
\tilde{\sigma} \\
-\vec{\sigma}\end{array}$ \\
\hline$\stackrel{x}{\sigma}$ & 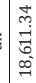 & 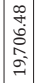 & 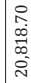 & 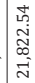 & 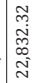 & $\mid \begin{array}{l}\infty \\
\tilde{\sigma} \\
\tilde{m} \\
\infty \\
\tilde{n} \\
\tilde{n}\end{array}$ & 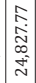 & $\mid \begin{array}{l}\mathfrak{1} \\
0 \\
0 \\
0 \\
2 \\
\end{array}$ & $\begin{array}{l}\hat{r} \\
\dot{\rho} \\
\hat{R} \\
\tilde{\delta}\end{array}$ & 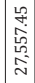 & $\left|\begin{array}{c}\mathfrak{w} \\
\dot{0} \\
\tilde{m} \\
\tilde{c} \\
\tilde{N}\end{array}\right|$ & 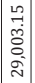 & 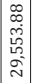 & 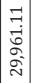 & 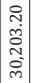 & 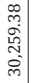 & 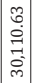 & 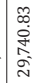 \\
\hline$\stackrel{x}{z}$ & 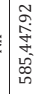 & 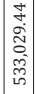 & $\begin{array}{l}\hat{0} \\
\dot{P} \\
\infty \\
0 \\
\tilde{\infty} \\
\infty \\
+\end{array}$ & 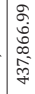 & 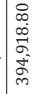 & 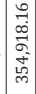 & 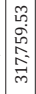 & 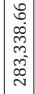 & 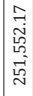 & 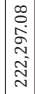 & 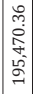 & 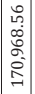 & 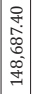 & 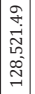 & 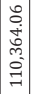 & 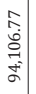 & & 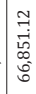 \\
\hline ă & 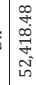 & 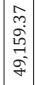 & 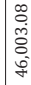 & 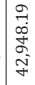 & $\begin{array}{l}\text { İ } \\
\dot{8} \\
\dot{8} \\
\dot{8}\end{array}$ & $\begin{array}{l}n \\
\infty \\
\infty \\
\omega \\
\tilde{\sim} \\
\tilde{\sim} \\
m\end{array}$ & 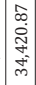 & 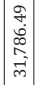 & 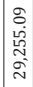 & 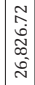 & 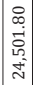 & 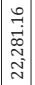 & 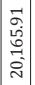 & 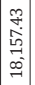 & 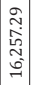 & 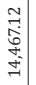 & 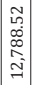 & 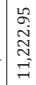 \\
\hline$\underline{\underline{\prime}}$ & 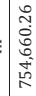 & 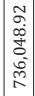 & 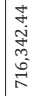 & 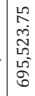 & 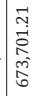 & $\begin{array}{l}0 \\
\infty \\
0 \\
0 \\
0 \\
0 \\
0 \\
0 \\
0\end{array}$ & $\begin{array}{l}\tilde{\sigma} \\
\tilde{\sigma} \\
\tilde{O} \\
\tilde{\tilde{D}} \\
\tilde{\sigma}\end{array}$ & 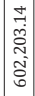 & 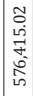 & 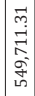 & 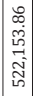 & 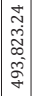 & 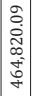 & 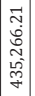 & 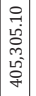 & 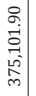 & 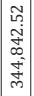 & 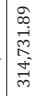 \\
\hline 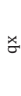 & 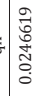 & $\mid \begin{array}{l}m \\
\stackrel{2}{\hat{~}} \\
\hat{0} \\
\tilde{0} \\
\dot{0}\end{array}$ & 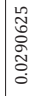 & $\begin{array}{l}\hat{n} \\
\text { m. } \\
\text { m. } \\
0 \\
0\end{array}$ & 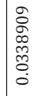 & 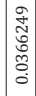 & 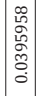 & 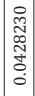 & 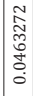 & $\begin{array}{l}\infty \\
0 \\
0 \\
0 \\
0 \\
0 \\
0 \\
0 \\
0\end{array}$ & 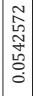 & $\begin{array}{l}\infty \\
\tilde{m} \\
\tilde{0} \\
\infty \\
0 \\
0 \\
0 \\
0\end{array}$ & $\begin{array}{l}m \\
\infty \\
\infty \\
0 \\
0 \\
0 \\
0 \\
0\end{array}$ & $\mid \begin{array}{c}0 \\
\stackrel{1}{1} \\
0 \\
0 \\
0 \\
0 \\
0 \\
0\end{array}$ & $\left|\begin{array}{c}0 \\
\vdots \\
⿱ 亠 䒑 \\
\vdots \\
\vdots \\
0 \\
0\end{array}\right|$ & $\begin{array}{l}\infty \\
\stackrel{0}{0} \\
\stackrel{0}{0} \\
\infty \\
0 \\
0 \\
0\end{array}$ & & 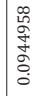 \\
\hline$x$ & $\mathscr{\bullet}$ & 8 & 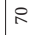 & 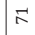 & 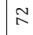 & $\Re$ & $\pi$ & $\stackrel{2}{\kappa}$ & $\pi$ & $\Sigma$ & $\stackrel{\infty}{\Re}$ & 2 & 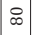 & $\vec{\infty}$ & $\approx$ & $\approx$ & $\varpi_{\infty}$ & $\not \infty$ \\
\hline
\end{tabular}
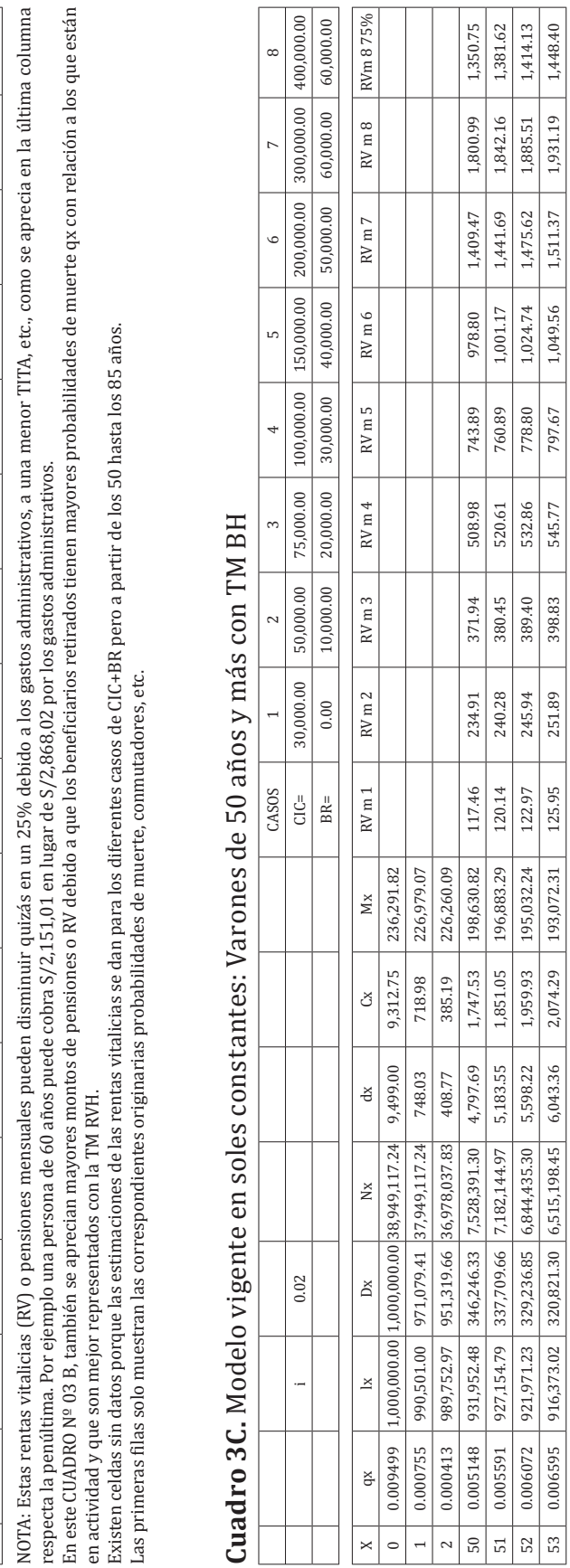


\begin{tabular}{|c|c|c|c|c|c|c|c|c|c|c|c|c|c|c|c|c|c|c|c|c|c|c|c|c|c|}
\hline & 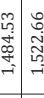 & 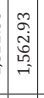 & 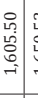 & 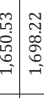 & 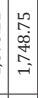 & $\begin{array}{c}0 \\
\tilde{2} \\
0 \\
0 \\
-i\end{array}$ & 空 & : & 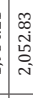 & 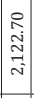 & 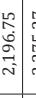 & 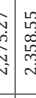 & 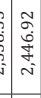 & 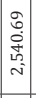 & 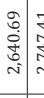 & 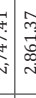 & 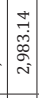 & $\begin{array}{c}m \\
m \\
\tilde{m} \\
\dot{m} \\
m \\
m\end{array}$ & 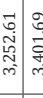 & 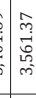 & 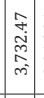 & $\vec{\sigma}$ & & & 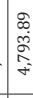 \\
\hline & 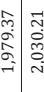 & 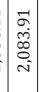 & 晜 & & $\begin{array}{c}\hat{\omega} \\
\dot{m} \\
m \\
\sim \\
\sim\end{array}$ & 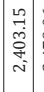 & 总 & 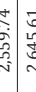 & 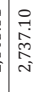 & 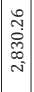 & $g$ & 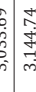 & 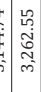 & 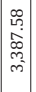 & 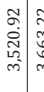 & 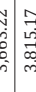 & 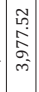 & 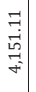 & 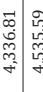 & 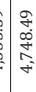 & 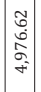 & is & i? & & 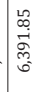 \\
\hline & Sid & 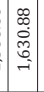 & & & 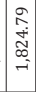 & $\begin{array}{l}\hat{m} \\
\hat{\infty} \\
\infty\end{array}$ & 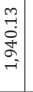 & 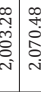 & 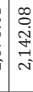 & 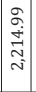 & 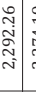 & $\frac{7}{7}$ & 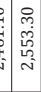 & 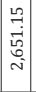 & 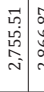 & 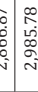 & 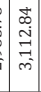 & 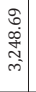 & 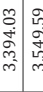 & స్త & 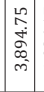 & + & 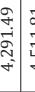 & & $\begin{array}{l}\text { స్ } \\
\text { ֻे } \\
\text { ஸे }\end{array}$ \\
\hline & $\begin{array}{l}0 \\
0 \\
0 \\
-1\end{array}$ & 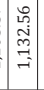 & & & 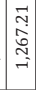 & 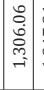 & 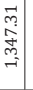 & 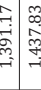 & 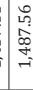 & 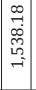 & $\infty$ & & 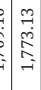 & 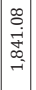 & 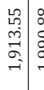 & 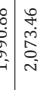 & 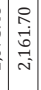 & 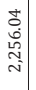 & 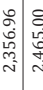 & 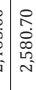 & 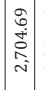 & i & & & लि \\
\hline 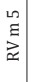 & $\begin{array}{l}\infty \\
\infty\end{array}$ & & & & & & & 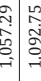 & 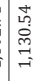 & $\mid$ & 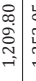 & 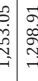 & & 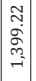 & & 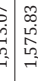 & 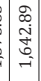 & | & & 胥 & & 宅 & & & \\
\hline & s. & $\begin{array}{l}\infty \\
\infty \\
\infty\end{array}$ & 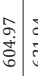 & - & & $\begin{array}{c}0 \\
\vdots \\
0 \\
6\end{array}$ & & & & & & & $\dot{i}$ & & & & ذ্ं & & & & \begin{tabular}{l}
7 \\
\multirow{8}{*}{}
\end{tabular} & & & & \\
\hline
\end{tabular}

m

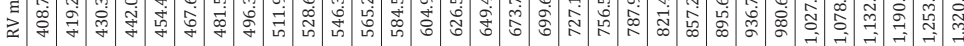

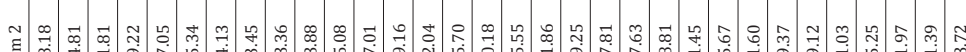

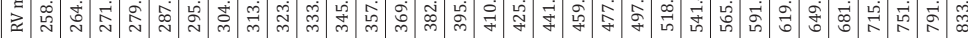

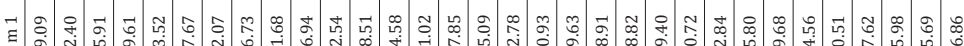

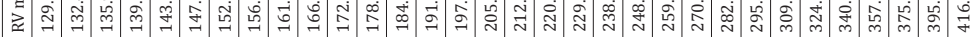

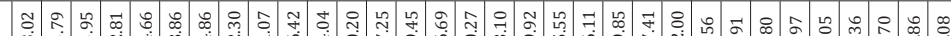

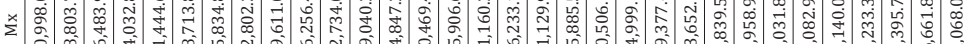

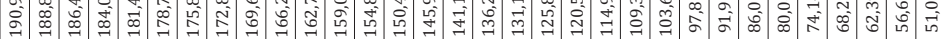
ๆ

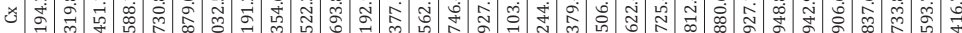

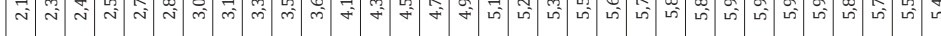
ำ

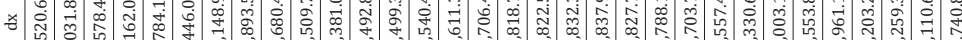

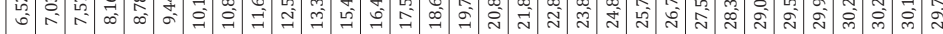
-

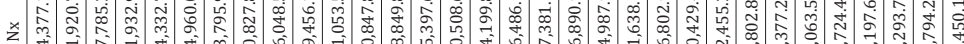

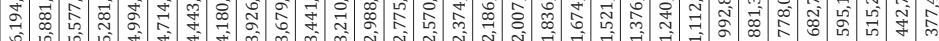

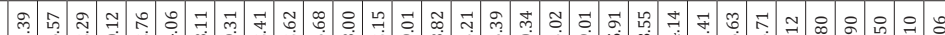

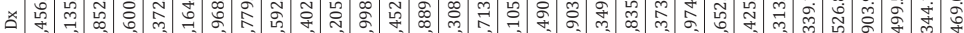

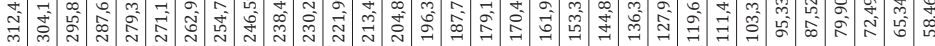
น น

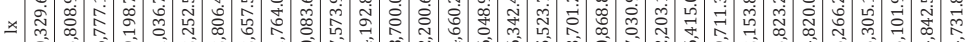

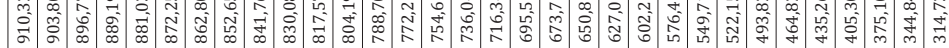

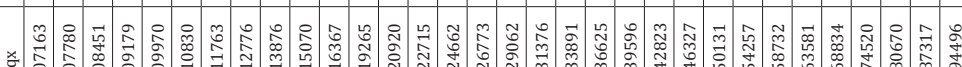

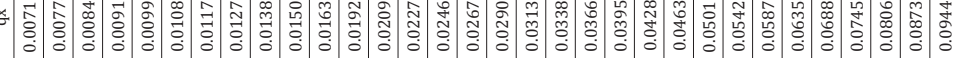
× ๖่

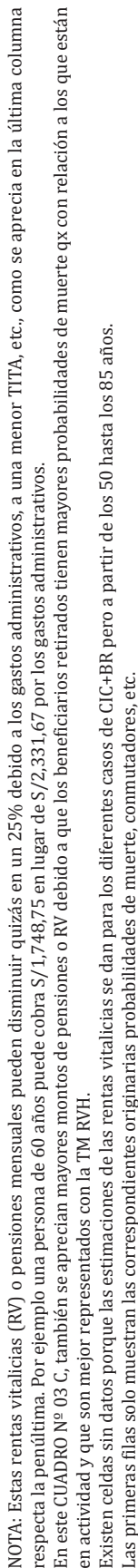


Pensamiento Crítico Vol. 23. N¹
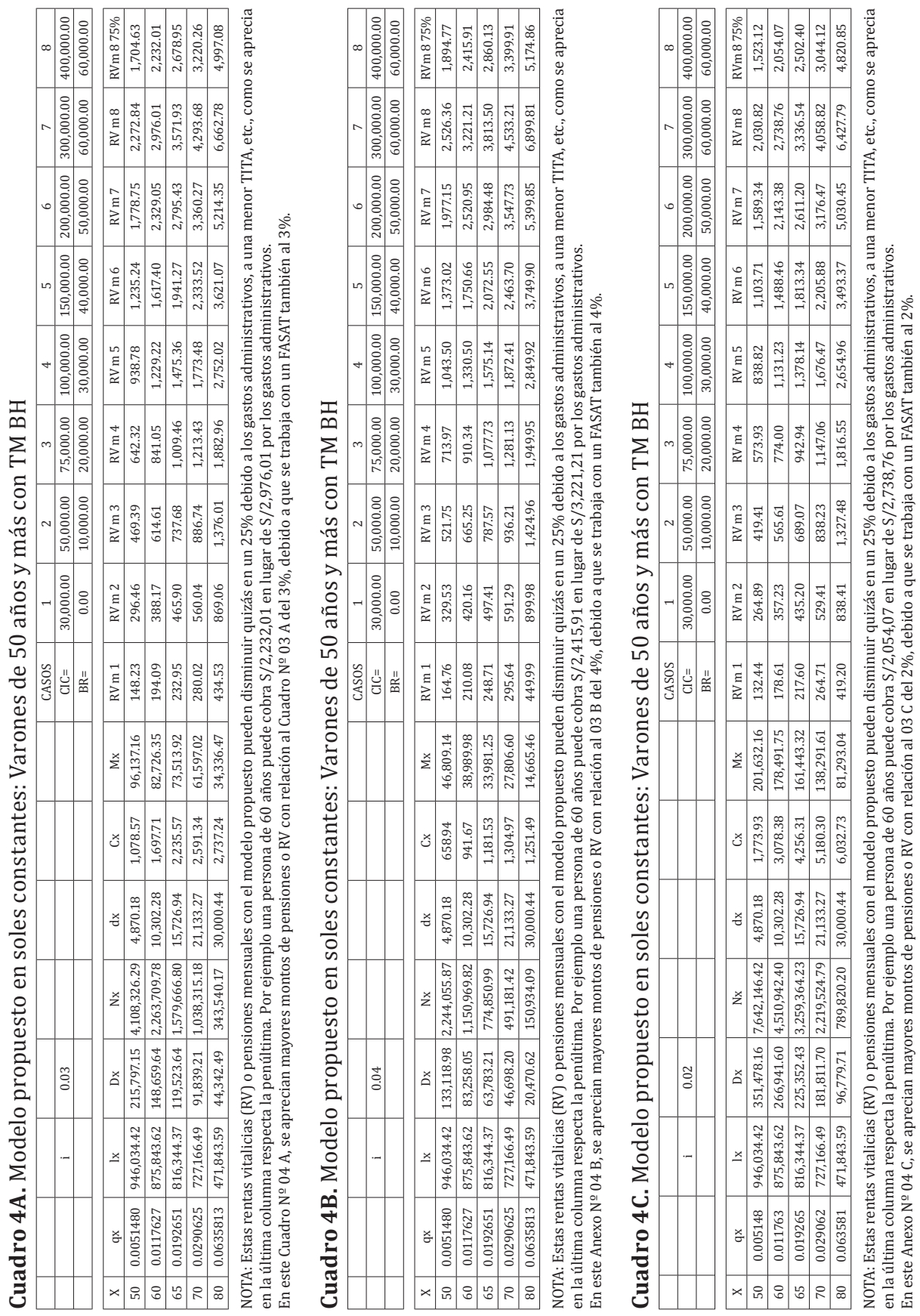


\section{Conclusiones}

Las siguientes conclusiones y otras, así como sus correspondientes recomendaciones, también se pueden apreciar en la tesis mencionada:

1. Según las Tablas de Mortalidad vigentes en el SPP, cada persona según su edad y sexo, vivirá de manera más probable un determinado número de años y no necesariamente hasta el límite de dichas tablas que es actualmente 110 años. Por ejemplo según la TM BH un varón de 65 años lo más probable es que viva 17.2 años más y no 45 años más hasta los 110 años y según la TM BM una mujer de 65 años vivirá 21.8 años más y no 45 años más. Es muy poco probable, tan solo $0.016 \%$, que un varón de 65 años llegue con vida a los 110 años, así como hay tan solo una probabilidad del $0.146 \%$ de que una mujer de 65 años llegue con vida a los 110 años. La mayoría de la población, por ejemplo los de 65 años, fallecerán alrededor de la edad estimada con su esperanza de vida.

2. Los modelos en general y los modelos actuariales en particular, por definición, deben trabajar y representar lo que ocurre en la realidad de la mejor manera posible, por ello es que en este trabajo, se considera que las personas vivirán de manera más probable hasta la edad definida por sus esperanzas de vida y no hasta los 110 años.

3. Los modelos actuariales propuestos logran mayor representatividad trabajando con las TM BH y BM que con las TM RVH y RVM, puesto que al contener mayores probabilidades de muerte representan mejor a las poblaciones de retirados, los que no solo por sus mayores edades sino también por aspectos vinculados al stress del cese, menor actividad en general, soledad, etc., generan efectivamente mayores probabilidades de muerte.

4. En los modelos elaborados, vigentes y propuestos, se comprueba la relación directa entre los montos de pensiones y las TITA empleadas y los fondos supuestos. 
5. También se verifica, para todos los casos, que al aumentar la edad de retiro, las RV también van aumentando debido a que ante un fondo hipotético constante, en cuanto la persona se retira de mayor edad, entonces tendrá menor esperanza de vida y dicho fondo, le alcanzará para generar una pensión más alta que comprende menos años de vida esperados.

6. Igual es verificable que en general, debido a que las mujeres tienen menores probabilidades de muerte que los hombres, entonces tienen mayores esperanzas de vida, son más longevas, teniéndosele que pagar por más tiempo sus RV, por lo que dichas $\mathrm{RV}$ resultan menores que las de los hombres, ante iguales fondos.

7. Se obtienen mayores montos de pensiones en los modelos propuestos de estimación de las RV no hasta el final de las TM sino estimadas hasta las fechas de fallecimiento más probables definidas por las esperanzas de vida de cada posible pensionista, según edades, sexos y las tablas vigentes disponibles.

8. Los cálculos de reservas matemáticas, al considerar el valor presente de las obligaciones para con los afiliados, al emplear cuando corresponda el modelo propuesto, generarán menores montos que los actuales cálculos, pues ya no se les pagaría, tanto a los pensionistas como a los que serán, hasta los 110 años, sino por un menor tiempo hasta cuando sus esperanzas de vida lo definan, disminuyendo en consecuencia el valor presente de las obligaciones.

9. En el SNP, DL 19990, Ley 20530, CPMP, etc., en donde las probabilidades de muerte en sus tablas son mayores que las del SPP, con el modelo propuesto se generarían menores esperanzas de vida, con menores reservas y menores impactos actuariales, económicos, financieros.

10. También se esperarían, con el criterio de las esperanzas de vida al cesar o adquirir derecho, mejoras en otros productos, prestaciones y beneficios en general, ofrecidos en la actual seguridad social. 
11. En la elaboración de los modelos en este trabajo se toma en cuenta siempre el concepto de equilibrio actuarial y sus bases técnicas o lo que es lo mismo la ecuación prestación contraprestación, los que son semejantes y se complementan con los criterios de los estudios económicos financieros.

\section{Recomendaciones}

1. Es conveniente considerar que la mayoría de las personas no vivirá hasta los 110 años, sino que fallecerán alrededor de la edad definida por su esperanza de vida con las Tablas de Mortalidad vigentes, existiendo para cada edad y sexo un determinado número de años que aún quedan por vivir de manera más probable.

2. Es muy importante recordar siempre en todo estudio, el concepto de modelo, es decir la representación más fidedigna o fehaciente de lo que ocurre o lo que va a ocurrir de manera más probable en el sistema o fenómeno estudiado, por ello es que en el presente trabajo y en otros semejantes se sugiere considerar que las personas vivirán de manera más probable hasta la edad definida por sus esperanzas de vida y no hasta los 110 años.

3. De las Tablas de Mortalidad vigentes, en el SPP, las más representativas y consistentes con el concepto de modelo en general y de modelo actuarial en particular para trabajar con los pensionistas, son las TM BH y BM que contienen mayores probabilidades de muerte con relación a las TM RVH y RVM que representan mejor a los afiliados en actividad.

4. Para la toma de decisiones del pensionista o de la empresa es bueno tener en cuenta la relación directa entre los montos de pensiones y las TITA empleadas y los fondos alcanzados.

5. También se debe tener en cuenta que a mayor edad de retiro, las RV también aumentan debido a que ante un fondo hipotético constante, en cuanto la persona se retira de mayor edad, entonces tendrá menor esperanza de vida y dicho fondo, le alcanzará para 
generar una pensión más alta que comprende menos años de vida esperados.

6. Es conveniente recordar que debido a que las mujeres tienen menores probabilidades de muerte que los hombres, entonces tienen mayores esperanzas de vida, son más longevas, teniéndosele que pagar por más tiempo sus RV, por lo que dichas $\mathrm{RV}$ resultan menores que las de los hombres, ante iguales fondos.

7. Es mejor trabajar en los modelos actuariales con el criterio de las esperanzas de vida pues no solo son modelos más representativos sino que también obtienen mayores montos de pensión de jubilación, viudez, ascendencia o invalidez.

8. Es bueno tener en mente que en alguna medida los mayores desembolsos que exigirían las mayores pensiones con el criterio de la esperanza de vida que se muestran en el presente trabajo y que podrían aplicarse a otras prestaciones, se compensarían con la mayor demanda por el mejor producto pensionario y porque los cálculos de reservas matemáticas, en los regímenes privados o públicos que correspondan, al considerar el valor presente de las obligaciones para con los afiliados, al emplear el modelo propuesto generarán menores montos que los actuales cálculos, pues ya no se les pagaría, tanto a los pensionistas como a los que serán, hasta los 110 años, sino por un menor tiempo hasta cuando sus esperanzas de vida lo definan, disminuyendo en consecuencia el valor presente de las obligaciones.

9. Se debe considerar que en los otros regímenes pensionarios en donde se constata mayores probabilidades de muerte que las existentes en el SPP, tales como en el SNP, DL 19990, Ley 20530, CPMP, mineros, etc., con el modelo propuesto se generarían menores esperanzas de vida al cese o adquirir el derecho, con menores reservas y menores impactos actuariales, económicos, financieros.

10. También conviene recordar que se esperarían, con el criterio de las esperanzas de vida al cesar o al adquirir el derecho, mejoras no solo en las pensiones de jubilación o vejez sino también en 
otros productos, prestaciones y beneficios en general, ofrecidos en la actual seguridad social, como son las pensiones de viudez, ascendencia e invalidez.

11. Así como en este trabajo, en otros semejantes como podrían ser los vinculados a la Reforma de la actual seguridad social, en especial la pensionaria u otras iniciativas, debieran tomarse en cuenta siempre los conceptos de esperanza de vida, modelo y equilibrio actuarial y sus bases técnicas, etc., los que son semejantes y se complementan con los criterios de los estudios económicos financieros. El obviar o minimizar su empleo, puede generar o seguir generando decisiones tomadas en modelos no representativos con sesgos políticos legales que no garantizan equilibrio.

\section{Referencias Bibliográficas}

Allen, Aníbal (1971). Matemática Actuarial, Lima. Editorial San Marcos. 136 p.

Anderson, Arthur (1992). Pension Mathematics for Actuaries. Connecticut. Editorial ACTEX Publications, Inc. USA.217 p.

Baca, Gabriel (1994). Fundamentos de Ingeniería Económica. México D.F, Editorial McGraw-Hill. 305 p.

Coppini, Mario (2002). Lezione Di Técnica delle Assicurazioni Sociali. CISU. 166 p. Fajardo, Martín (1985). Derecho de la Seguridad Social, Lima. M. Fajardo C. 275 p.

González Galé, José (1977). Elementos de Cálculo Actuarial. Buenos Aires. Ediciones Macchi. 287 p.

Lasheras, Antonio (1948). Matemática del Seguro. Madrid. Editorial Dossat, S.A.690 páginas.

Palacios, Hugo (1996). Introducción al Cálculo Actuarial. Madrid. Editorial MAPFRE, S.A. 197 p.

Tarquin, Anthony (1978). Ingeniería Económica. México D. F. Editorial McGrawHill. 412 p.

Taylor, George (1978). Ingeniería Económica. México D. F. Editorial Limusa S. A. $556 \mathrm{p}$. 
Pensamiento Crítico Vol. 23. $\mathbf{N}^{\circ} 1$

Thullen, Peter (1973). Manuel des Techniques Actuarielles delle SécuritéSociale, México D. F. Editorial OIT. 462 p.

Thullen, Peter (1995). Técnicas Actuariales de la Seguridad Social. México D. F. Editorial OIT. $532 \mathrm{p}$. 
